\title{
Nature of the Trigger Linkage in Explosive Materials Is a Charge-Shift Bond
}

\author{
Jyothish Joy, * David Danovich, and Sason Shaik* \\ Institute of Chemistry, The Hebrew University of Jerusalem, Jerusalem, 91904, Israel \\ * Corresponding authors E-mail: sason@yfaat.ch.huji.ac.il; jyothish.joy@mail.huji.ac.il.
}

\section{Table of Contents}

Geometrical features and electrostatic potential map of the model compounds S2

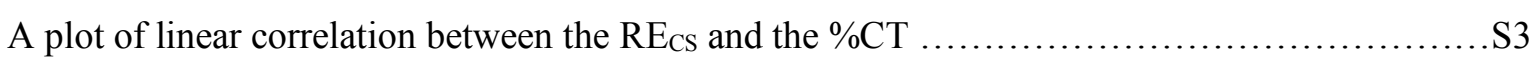

Correlation between Laplacian of electron density and the shock sensitivity $\left(\ln \mathrm{P}_{\text {expt }}\right) \ldots \ldots \ldots \ldots . . . . . .53$

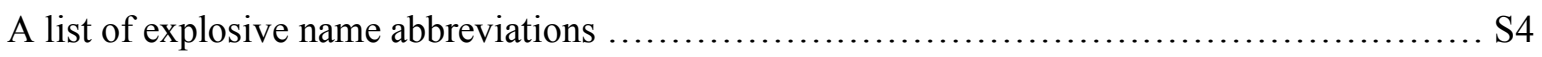

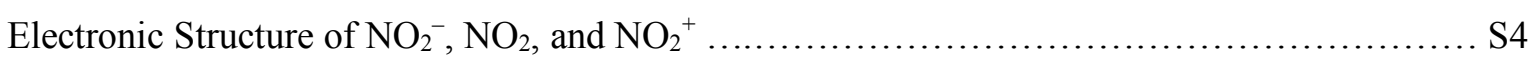

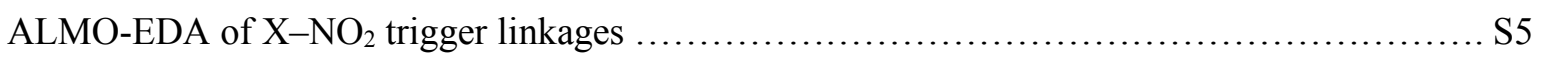

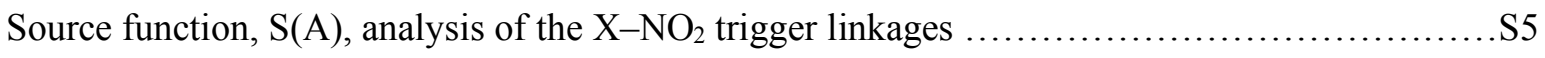

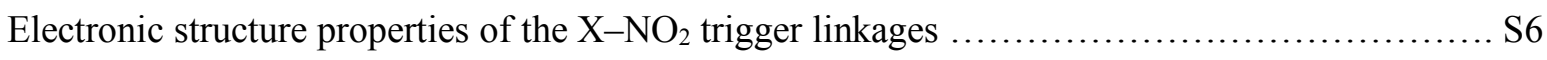

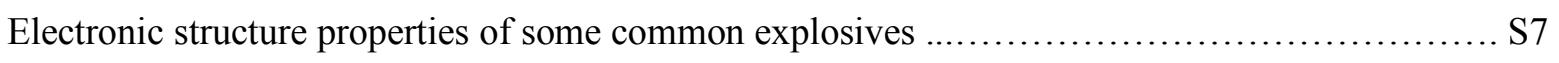

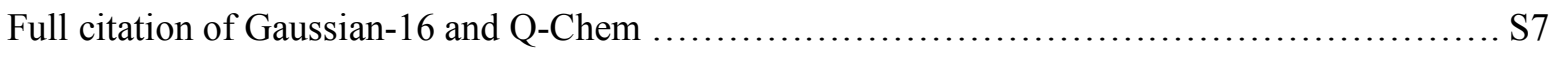

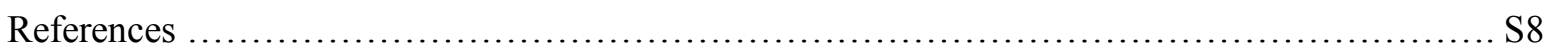

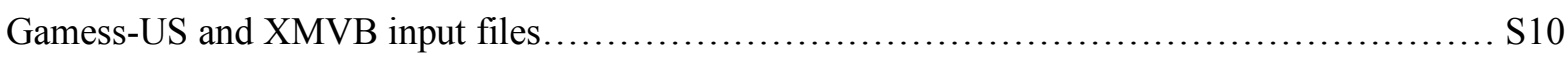

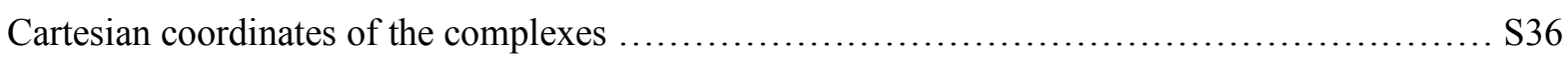




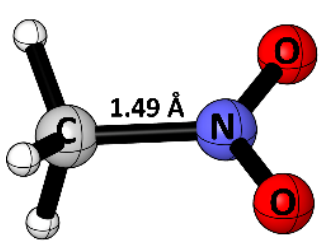

$\mathrm{H}_{3} \mathrm{C}-\mathrm{NO}_{2}$

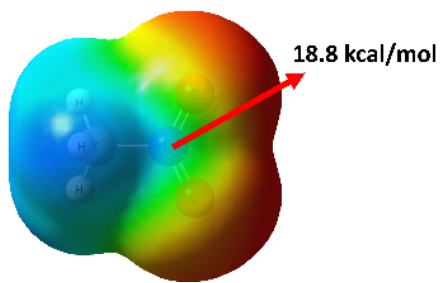

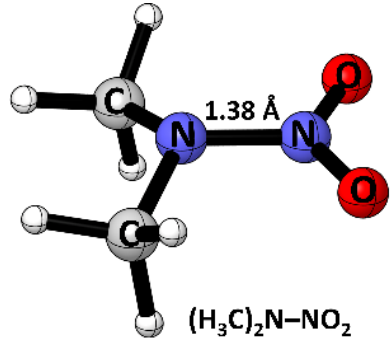

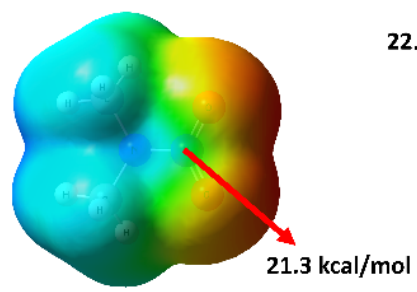

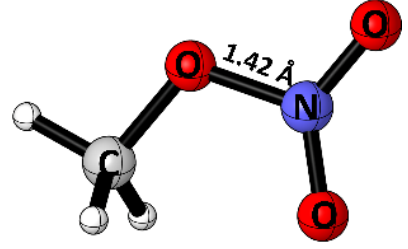

$\left(\mathrm{H}_{3} \mathrm{C}\right) \mathrm{O}-\mathrm{NO}_{2}$

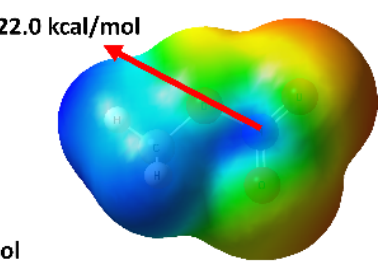

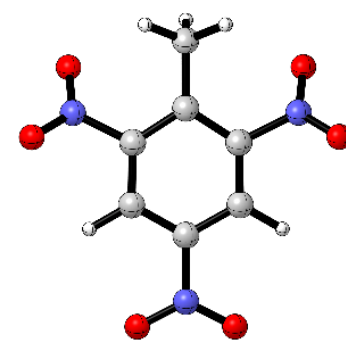

TNT

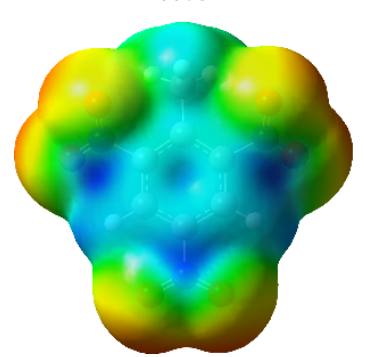

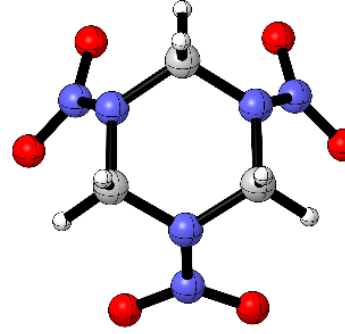

RDX

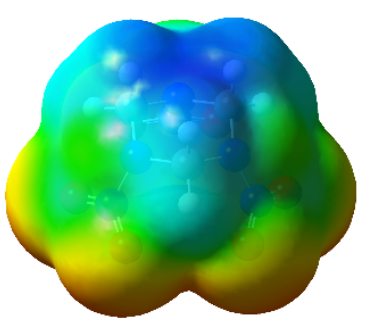

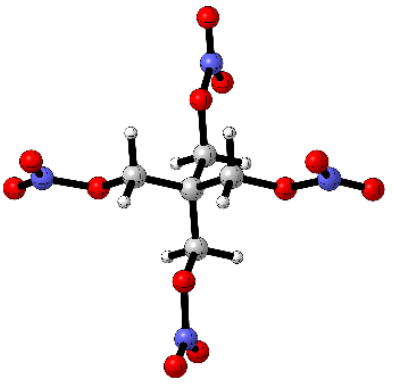

PETN

Figure S1. Geometrical features and electrostatic potential (ESP) map of the model compounds and their realistic counterparts (TNT, RDX, and PETN) calculated at the B2PLYP-D3/cc-pVTZ level of theory. Red- and blue- color indicates regions of negative and positive ESP values respectively. The uneven charge distribution over the $\mathrm{X}-\mathrm{N}$ bond illustrates the extent of charge imbalance experienced by the trigger linkage that correlates with its sensitivity to impact. The larger the charge imbalance, the higher is the impact sensitivity. ${ }^{\text {S1-S4 }}$ 


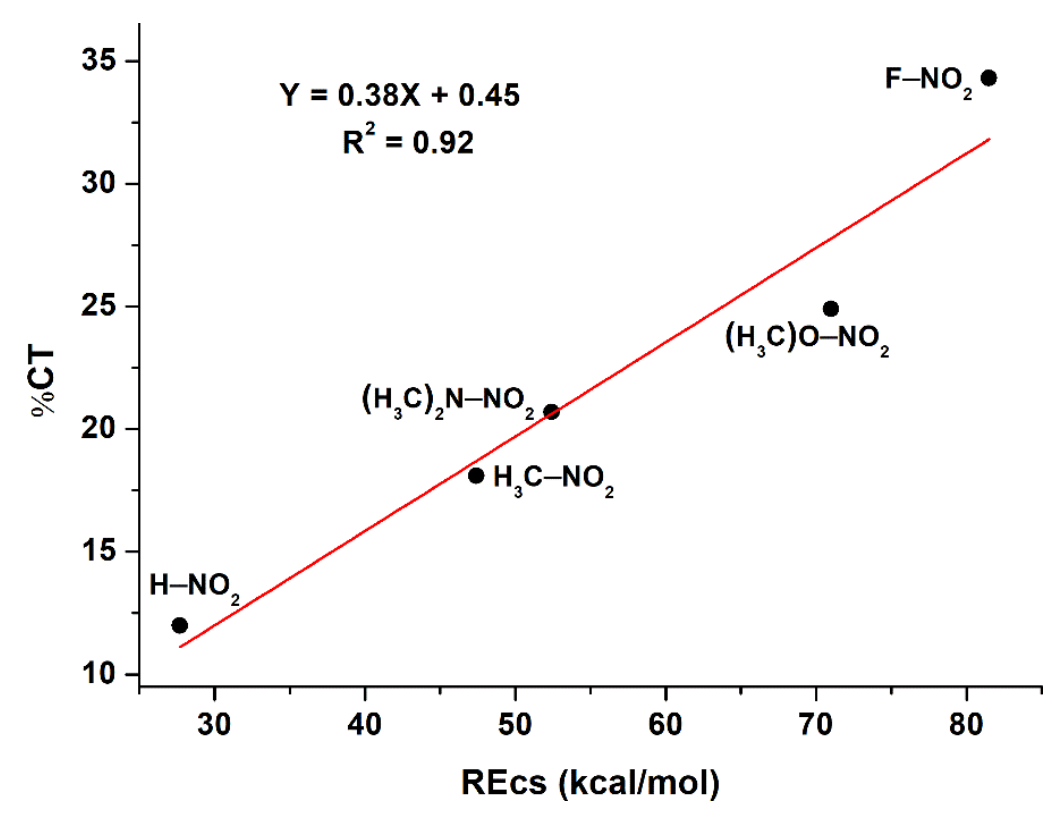

Figure S2. A plot showing the linear correlation between the REcs from D-BOVB and the \%CT from ALMO-EDA calculations.

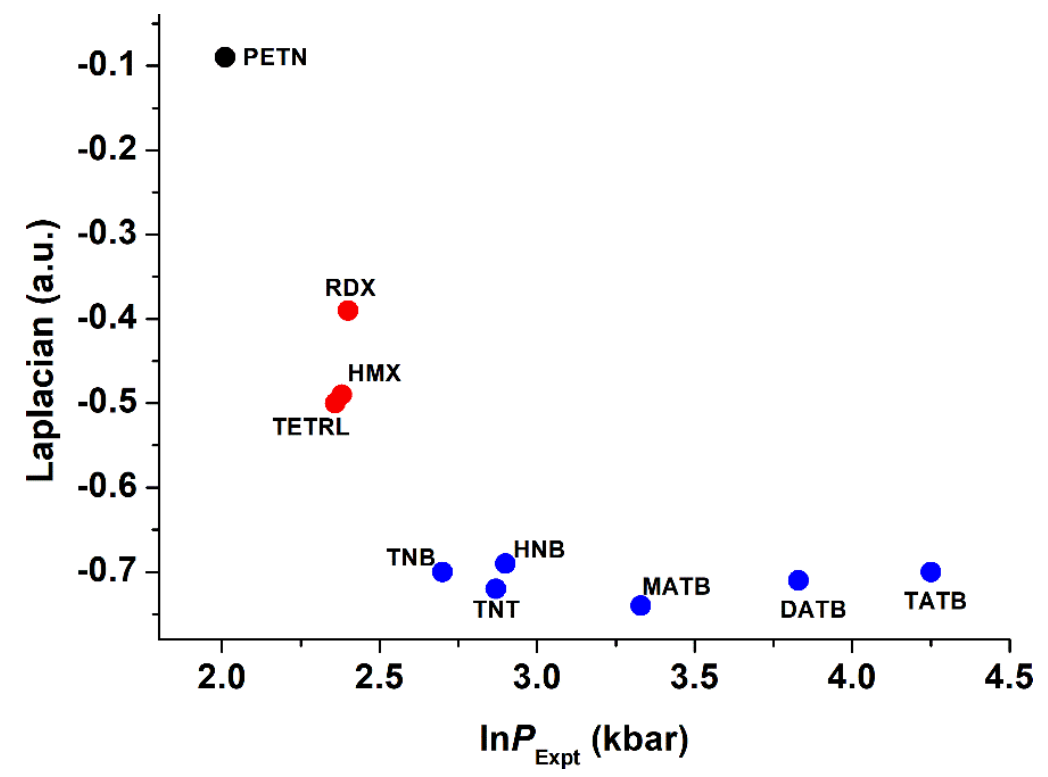

Figure S3. Correlation between the Laplacian of electron density at the $\mathrm{X}-\mathrm{NO}_{2}$ bond critical point and the natural logarithm of shock sensitivity $\left(\ln \mathrm{P}_{\text {expt }}\right)$ for a selected set of explosive molecules belonging to $\mathrm{C}-\mathrm{NO}_{2}$ (blue), $\mathrm{N}-\mathrm{NO}_{2}$ (red), and $\mathrm{O}-\mathrm{NO}_{2}$ (black) trigger linkages. The experimental shock sensitivity is taken from the work of Storm et al. ${ }^{\mathrm{S} 5}$ 


\section{A list of explosive name abbreviations:}

(1) PETN = Tetranitrate Pentaerythritol

(2) $\mathrm{RDX}=1,3,5$-Trinitroperhydro-1,3,5-Triazine

(3) $\mathrm{HMX}=1,3,5,7$-Tetranitro-1,3,5,7-Tetraazacyclooctane

(4) TETRYL $=2,4,6$-Trinitrophenylmethylnitramine

(5) $\mathrm{TNT}=2,4,6$-Trinitrotoluene

(6) $\mathrm{TNB}=1,3,5$-Trinitrobenzene

(7) $\mathrm{HNB}=$ Hexanitrobenzene

(8) MATB = Monoamino-2,4,6-Trinitrobenzene

(9) $\mathrm{DATB}=$ 1,3-Diamino-2,4,6-Trinitrobenzene

(10) TATB $=1,3,5$-Triamino-2,4,6-Trinitrobenzene

\section{Electronic Structure of $\mathrm{NO}_{2}^{-}, \mathrm{NO}_{2}$, and $\mathrm{NO}_{2}{ }^{+}$}

The Lewis representation and geometrical features of $\mathrm{NO}_{2}{ }^{-}, \mathrm{NO}_{2}$, and $\mathrm{NO}_{2}{ }^{+}$molecules are displayed in Figure S4. As we move from $\mathrm{NO}_{2}^{-}$to $\mathrm{NO}_{2}{ }^{+}$, the $\mathrm{N}-\mathrm{O}$ bond length decreases while the $\mathrm{O}-\mathrm{N}-\mathrm{O}$ bond angle increases, and these changes occur in accord with their Lewis representations. Furthermore, the central nitrogen atom in all three cases is positively charged irrespective of their overall charge.
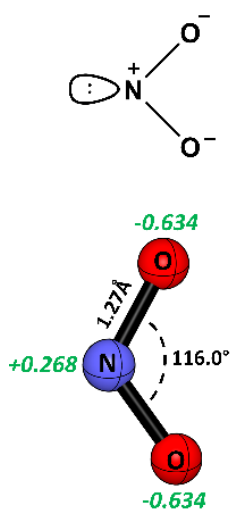

(a) $\mathrm{NO}_{2}^{-}$<smiles></smiles>

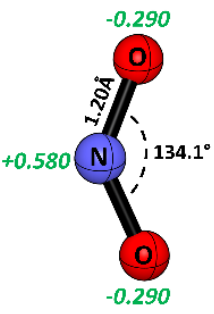

(b) $\mathrm{NO}_{2}$
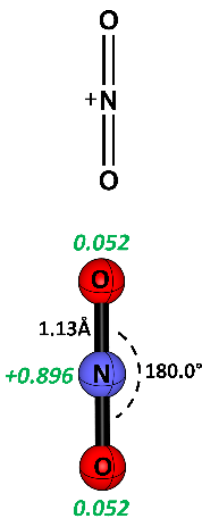

(c) $\mathrm{NO}_{2}^{+}$

Figure S4. Pictorial representation of the Lewis structures and their corresponding geometric parameters such as $\mathrm{N}-\mathrm{O}$ bond length and $\mathrm{O}-\mathrm{N}-\mathrm{O}$ bond angle for (a) $\mathrm{NO}_{2}^{-}$, (b) $\mathrm{NO}_{2}$, and (c) $\mathrm{NO}_{2}{ }^{+}$. Natural charges of the $\mathrm{N}$ and $\mathrm{O}$ atoms from NBO analysis are shown in green italic numbers. 
Table S1. ALMO-EDA of $\mathrm{X}-\mathrm{NO}_{2}$ trigger linkages. Calculations are done at the $\omega \mathrm{B} 97 \mathrm{M}-\mathrm{V} /$ aug-ccpVTZ level of theory. All energies are given in $\mathrm{kcal} / \mathrm{mol}$. Values in parentheses are the percentages of the total stabilizing interaction energy. For a detailed description of the energy terms refer to Levine et al., Proc. Natl. Acad. Sci. U. S. A. 2017, 114, 12649-12656.

\begin{tabular}{|c|c|c|c|c|c|c|c|}
\hline $\begin{array}{l}\text { Trigger } \\
\text { Linkage }\end{array}$ & $\operatorname{PREP}_{(\mathrm{Geom})}$ & $\operatorname{PREP}_{\text {(Elect) }}$ & FRZ & $\mathrm{SC}$ & POL & CT & Sum \\
\hline $\mathrm{H}-\mathrm{NO}_{2}$ & 2.52 & 24.74 & 263.37 & $\begin{array}{c}-252.97 \\
(68.67 \%)\end{array}$ & $\begin{array}{c}-71.37 \\
(19.4 \%)\end{array}$ & $\begin{array}{c}-44.33 \\
(12.0 \%)\end{array}$ & -78.04 \\
\hline $\mathrm{H}_{3} \mathrm{C}-\mathrm{NO}_{2}$ & 9.58 & 40.03 & 262.51 & $\begin{array}{l}-256.40 \\
(67.3 \%)\end{array}$ & $\begin{array}{c}-55.80 \\
(14.6 \%)\end{array}$ & $\begin{array}{c}-68.89 \\
(18.1 \%)\end{array}$ & -68.97 \\
\hline$\left(\mathrm{H}_{3} \mathrm{C}\right)_{2} \mathrm{~N}-\mathrm{NO}_{2}$ & 8.92 & 74.08 & 343.62 & $\begin{array}{l}-302.29 \\
(62.9 \%)\end{array}$ & $\begin{array}{c}-79.17 \\
(16.5 \%)\end{array}$ & $\begin{array}{c}-99.41 \\
(20.7 \%)\end{array}$ & -54.25 \\
\hline$\left(\mathrm{H}_{3} \mathrm{C}\right) \mathrm{O}-\mathrm{NO}_{2}$ & 3.75 & 40.61 & 261.09 & $\begin{array}{l}-202.56 \\
(57.4 \%)\end{array}$ & $\begin{array}{c}-62.36 \\
(17.7 \%)\end{array}$ & $\begin{array}{c}-87.89 \\
(24.9 \%)\end{array}$ & -47.36 \\
\hline $\mathrm{F}-\mathrm{NO}_{2}$ & 0.97 & 14.37 & 173.86 & $\begin{array}{l}-116.80 \\
(46.8 \%)\end{array}$ & $\begin{array}{c}-47.16 \\
(18.9 \%)\end{array}$ & $\begin{array}{c}-85.54 \\
(34.3 \%)\end{array}$ & -60.30 \\
\hline
\end{tabular}

\section{Source Function Analysis of the Trigger Linkages}

A very useful analysis to gauge the importance of negative hyperconjugation is of the source function, $\mathrm{S}(\mathrm{A}),{ }^{\mathrm{S} 6-\mathrm{S} 7}$ of electron density at the $\mathrm{X}-\mathrm{NO}_{2}$ bond critical point. $\mathrm{S}(\mathrm{A})$ provides information about the involvement of oxygen lone-pairs into the trigger linkage and quantifies the individual atomic contributions to the total electron density at the $\mathrm{X}-\mathrm{NO}_{2}$ bond critical point. Our $\mathrm{S}(\mathrm{A})$ analysis (Table S2) of the trigger linkages shows that the $\mathrm{N}$-atom of $\mathrm{X}-\mathrm{NO}_{2}$ donates $30-37 \%$ of electron density to the $\mathrm{X}-\mathrm{N}$ bond critical point, while the rest, $12-20 \%$, is coming from the oxygen atoms of the $\mathrm{NO}_{2}$ group. As the group electronegativity of X-atom/group increases, the contribution from nitrogen decreases, and that from the oxygens increases, ultimately weakening the $\mathrm{X}-\mathrm{NO}_{2}$ trigger linkage. Overall, our $\mathrm{S}(\mathrm{A})$ analysis directly point towards the decisive role of oxygen lone-pairs on the CSB nature of the $\mathrm{X}-\mathrm{NO}_{2}$ trigger linkage via negative hyperconjugation. 
Table S2. Source function, $\mathrm{S}(\mathrm{A})$, analysis of the $\mathrm{X}-\mathrm{NO}_{2}$ trigger linkages. $\rho_{(r)}$ is the electron density at the $\mathrm{X}-\mathrm{NO}_{2}$ bond critical point. $\mathrm{S} \%$ represents the percentage contribution of individual atoms/fragments to the electron density $\rho_{(r)}$.

\begin{tabular}{|c|c|c|c|c|}
\hline $\mathrm{X}-\mathrm{NO}_{2}$ & $\begin{array}{c}\rho_{(r)} \\
\text { (a.u.) }\end{array}$ & $\begin{array}{c}\mathrm{S} \%(\mathrm{~N}) \\
(\%)\end{array}$ & $\begin{array}{c}\mathrm{S} \%(\mathrm{O}) \\
(\%)\end{array}$ & $\begin{array}{c}\mathrm{S} \%(\mathrm{X}) \\
(\%)\end{array}$ \\
\hline $\mathrm{H}-\mathrm{NO}_{2}$ & 0.34 & 45.6 & 11.9 & 42.5 \\
\hline $\mathrm{H}_{3} \mathrm{C}-\mathrm{NO}_{2}$ & 0.25 & 33.9 & 12.8 & 53.3 \\
\hline$\left(\mathrm{H}_{3} \mathrm{C}\right)_{2} \mathrm{~N}-\mathrm{NO}_{2}$ & 0.36 & 36.7 & 12.1 & 51.2 \\
\hline$\left(\mathrm{H}_{3} \mathrm{C}\right) \mathrm{O}-\mathrm{NO}_{2}$ & 0.32 & 34.0 & 15.0 & 51.0 \\
\hline $\mathrm{F}-\mathrm{NO}_{2}$ & 0.25 & 30.2 & 19.9 & 49.9 \\
\hline
\end{tabular}

Table S3. Electronic structure properties of the $\mathrm{X}-\mathrm{NO}_{2}$ trigger linkages. The bond dissociation energy (BDE), charge-shift resonance energy (REcs), and the magnitude of negative hyperconjugation (NegHyp) in $\mathrm{kcal} / \mathrm{mol}$. The O-N-O angle in the $\mathrm{NO}_{2}$ group in degrees unit $(\angle \mathrm{O}-\mathrm{N}-\mathrm{O})$. Electron density $\left(\rho_{(r)}\right)$ and its Laplacian $\left(\nabla^{2} \rho_{(r)}\right)$ at the $\mathrm{X}-\mathrm{NO}_{2}$ bond critical point in atomic units. BDE and REcs are obtained from D-BOVB/cc-pVDZ level of theory.

\begin{tabular}{|c|c|c|c|c|c|c|}
\hline $\mathrm{X}-\mathrm{NO}_{2}$ & $\begin{array}{c}\mathrm{BDE} \\
(\mathrm{kcal} / \mathrm{mol})\end{array}$ & $\begin{array}{c}\mathrm{REcs} \\
(\mathrm{kcal} / \mathrm{mol})\end{array}$ & $\begin{array}{c}\mathrm{NegHyp} \\
(\mathrm{kcal} / \mathrm{mol})\end{array}$ & $\begin{array}{c}\angle \mathrm{O}-\mathrm{N}-\mathrm{O} \\
(\text { degree })\end{array}$ & $\begin{array}{c}\rho_{(r)} \\
(\text { a.u. })\end{array}$ & $\begin{array}{c}\nabla^{2} \rho_{(r)} \\
\text { (a.u. })\end{array}$ \\
\hline $\mathrm{H}-\mathrm{NO}_{2}$ & 75.0 & 27.7 & 30.9 & 128.4 & 0.34 & -1.90 \\
\hline $\mathrm{H}_{3} \mathrm{C}-\mathrm{NO}_{2}$ & 69.7 & 47.4 & 30.6 & 125.9 & 0.25 & -0.70 \\
\hline$\left(\mathrm{H}_{3} \mathrm{C}\right)_{2} \mathrm{~N}-\mathrm{NO}_{2}$ & 51.6 & 52.4 & 43.2 & 126.0 & 0.36 & -0.66 \\
\hline$\left(\mathrm{H}_{3} \mathrm{C}\right) \mathrm{O}-\mathrm{NO}_{2}$ & 47.4 & 71.0 & 75.1 & 130.2 & 0.32 & -0.25 \\
\hline $\mathrm{F}-\mathrm{NO}_{2}$ & 51.5 & 81.5 & 138.9 & 136.3 & 0.25 & +0.01 \\
\hline
\end{tabular}


Table S4. Electronic structure properties of the trigger linkages in model complexes of some of the common explosive materials. The bond dissociation energy (BDE), it's covalent component (BDE $\left.{ }_{\mathrm{Cov}}\right)$, and charge-shift resonance energy (REcs) are in $\mathrm{kcal} / \mathrm{mol}$. \%REcs is the percentage of charge-shift character to the total $\mathrm{BDE}\left[\% \mathrm{RE}_{\mathrm{CS}}=\left(\mathrm{RE}_{\mathrm{CS}} / \mathrm{BDE}\right) \times 100\right]$. Electron density $\left(\rho_{(r)}\right)$ and its Laplacian $\left(\nabla^{2} \rho_{(r)}\right)$ at the trigger linkage in atomic units.

\begin{tabular}{|c|c|c|c|c|c|c|}
\hline Explosive & $\begin{array}{c}\mathrm{BDE} \\
(\mathrm{kcal} / \mathrm{mol})\end{array}$ & $\begin{array}{c}\mathrm{BDE} \text { (ov } \\
(\mathrm{kcal} / \mathrm{mol})\end{array}$ & $\begin{array}{c}\mathrm{REcs} \\
(\mathrm{kcal} / \mathrm{mol})\end{array}$ & $\begin{array}{c}\% \mathrm{REcs} \\
(\%)\end{array}$ & $\begin{array}{c}\rho_{(r)} \\
(\text { a.u. })\end{array}$ & $\begin{array}{c}\nabla^{2} \rho_{(r)} \\
(\text { a.u. })\end{array}$ \\
\hline $\begin{array}{c}\text { Peroxide } \\
\mathrm{HO}-\mathrm{OH}^{\mathrm{a}}\end{array}$ & 49.8 & -7.1 & 56.9 & 114.3 & 0.26 & -0.02 \\
\hline $\begin{array}{c}\text { Nitrate } \\
\mathrm{O}_{2} \mathrm{~N}-\mathrm{OH}\end{array}$ & 58.6 & 4.0 & 54.6 & 93.2 & 0.32 & -0.17 \\
\hline $\begin{array}{c}\text { Perchlorate } \\
\mathrm{O}_{3} \mathrm{Cl}-\mathrm{OH}\end{array}$ & 55.8 & 16.6 & 39.2 & 70.2 & 0.21 & -0.12 \\
\hline $\begin{array}{c}\text { Iodides } \\
\mathrm{I}_{2} \mathrm{~N}-\mathrm{I}\end{array}$ & 25.4 & 5.2 & 20.2 & 79.5 & 0.11 & +0.70 \\
\hline
\end{tabular}

${ }^{a}$ Data taken from reference S8.

\section{Full citation of Gaussian-16}

Frisch, M. J.; Trucks, G. W.; Schlegel, H. B.; Scuseria, G. E.; Robb, M. A.; Cheeseman, J. R.; Scalmani, G.; Barone, V.; Petersson, G. A.; Nakatsuji, H.; Li, X.; Caricato, M.; Marenich, A. V.; Bloino, J.; Janesko, B. G.; Gomperts, R.; Mennucci, B.; Hratchian, H. P.; Ortiz, J. V.; Izmaylov, A. F.; Sonnenberg, J. L.; Williams; Ding, F.; Lipparini, F.; Egidi, F.; Goings, J.; Peng, B.; Petrone, A.; Henderson, T.; Ranasinghe, D.; Zakrzewski, V. G.; Gao, J.; Rega, N.; Zheng, G.; Liang, W.; Hada, M.; Ehara, M.; Toyota, K.; Fukuda, R.; Hasegawa, J.; Ishida, M.; Nakajima, T.; Honda, Y.; Kitao, O.; Nakai, H.; Vreven, T.; Throssell, K.; Montgomery Jr., J. A.; Peralta, J. E.; Ogliaro, F.; Bearpark, M. J.; Heyd, J. J.; Brothers, E. N.; Kudin, K. N.; Staroverov, V. N.; Keith, T. A.; Kobayashi, R.; Normand, J.; Raghavachari, K.; Rendell, A. P.; Burant, J. C.; Iyengar, S. S.; Tomasi, J.; Cossi, M.; Millam, J. M.; Klene, M.; Adamo, C.; Cammi, R.; Ochterski, J. W.; Martin, R. L.; Morokuma, K.; Farkas, O.; Foresman, J. B.; Fox, D. J. Gaussian 16 Rev. B.01, Wallingford, CT, 2016. 


\section{Full citation of Q-Chem}

Shao, Y.; Gan, Z.; Epifanovsky, E.; Gilbert, A. T. B.; Wormit, M.; Kussmann, J.; Lange, A. W.; Behn, A.; Deng, J.; Feng, X.; Ghosh, D.; Goldey, M.; Horn, P. R.; Jacobson, L. D.; Kaliman, I.; Khaliullin, R. Z.; Kuś, T.; Landau, A.; Liu, J.; Proynov, E. I.; Rhee, Y. M.; Richard, R. M.; Rohrdanz, M. A.; Steele, R. P.; Sundstrom, E. J.; Woodcock, H. L.; Zimmerman, P. M.; Zuev, D.; Albrecht, B.; Alguire, E.; Austin, B.; Beran, G. J. O.; Bernard, Y. A.; Berquist, E.; Brandhorst, K.; Bravaya, K. B.; Brown, S. T.; Casanova, D.; Chang, C.-M.; Chen, Y.; Chien, S. H.; Closser, K. D.; Crittenden, D. L.; Diedenhofen, M.; DiStasio, R. A.; Do, H.; Dutoi, A. D.; Edgar, R. G.; Fatehi, S.; Fusti-Molnar, L.; Ghysels, A.; Golubeva-Zadorozhnaya, A.; Gomes, J.; Hanson-Heine, M. W. D.; Harbach, P. H. P.; Hauser, A. W.; Hohenstein, E. G.; Holden, Z. C.; Jagau, T.-C.; Ji, H.; Kaduk, B.; Khistyaev, K.; Kim, J.; Kim, J.; King, R. A.; Klunzinger, P.; Kosenkov, D.; Kowalczyk, T.; Krauter, C. M.; Lao, K. U.; Laurent, A. D.; Lawler, K. V.; Levchenko, S. V.; Lin, C. Y.; Liu, F.; Livshits, E.; Lochan, R. C.; Luenser, A.; Manohar, P.; Manzer, S. F.; Mao, S.-P.; Mardirossian, N.; Marenich, A. V.; Maurer, S. A.; Mayhall, N. J.; Neuscamman, E.; Oana, C. M.; Olivares-Amaya, R.; O’Neill, D. P.; Parkhill, J. A.; Perrine, T. M.; Peverati, R.; Prociuk, A.; Rehn, D. R.; Rosta, E.; Russ, N. J.; Sharada, S. M.; Sharma, S.; Small, D. W.; Sodt, A.; Stein, T.; Stück, D.; Su, Y.-C.; Thom, A. J. W.; Tsuchimochi, T.; Vanovschi, V.; Vogt, L.; Vydrov, O.; Wang, T.; Watson, M. A.; Wenzel, J.; White, A.; Williams, C. F.; Yang, J.; Yeganeh, S.; Yost, S. R.; You, Z.-Q.; Zhang, I. Y.; Zhang, X.; Zhao, Y.; Brooks, B. R.; Chan, G. K. L.; Chipman, D. M.; Cramer, C. J.; Goddard, W. A.; Gordon, M. S.; Hehre, W. J.; Klamt, A.; Schaefer, H. F.; Schmidt, M. W.; Sherrill, C. D.; Truhlar, D. G.; Warshel, A.; Xu, X.; Aspuru-Guzik, A.; Baer, R.; Bell, A. T.; Besley, N. A.; Chai, J.-D.; Dreuw, A.; Dunietz, B. D.; Furlani, T. R.; Gwaltney, S. R.; Hsu, C.-P.; Jung, Y.; Kong, J.; Lambrecht, D. S.; Liang, W.; Ochsenfeld, C.; Rassolov, V. A.; Slipchenko, L. V.; Subotnik, J. E.; Van Voorhis, T.; Herbert, J. M.; Krylov, A. I.; Gill, P. M. W.; Head-Gordon, M. Advances in molecular quantum chemistry contained in the Q-Chem 4 program package. Mol. Phys. 2015, 113, 184-215.

\section{$\underline{\text { References }}$}

S1. Murray, J. S.; Lane, P.; Politzer, P. Relationships between impact sensitivities and molecular surface electrostatic potentials of nitroaromatic and nitroheterocyclic molecules. Mol. Phys. 1995, 85, $1-8$.

S2. Jane S. Murray Pat Lane Peter, P. Effects of strongly electron-attracting components on molecular surface electrostatic potentials: application to predicting impact sensitivities of energetic molecules. Mol. Phys. 1998, 93, 187-194. 
S3. Murray, J. S.; Concha, M. C.; Politzer, P. Links between surface electrostatic potentials of energetic molecules, impact sensitivities and $\mathrm{C}-\mathrm{NO}_{2} / \mathrm{N}-\mathrm{NO}_{2}$ bond dissociation energies. Mol. Phys. 2009, 107, 89-97.

S4. Rice, B. M.; Hare, J. J. A Quantum Mechanical Investigation of the Relation between Impact Sensitivity and the Charge Distribution in Energetic Molecules. J. Phys. Chem. A 2002, 106, 17701783.

S5. Storm, C. B.; Stine, J. R.; Kramer, J. F., Sensitivity Relationships in Energetic Materials. In Chemistry and Physics of Energetic Materials, Bulusu, S. N., Ed. Springer Netherlands: Dordrecht, 1990; pp 605-639.

S6. Bader, R. F. W.; Gatti, C. A Green's function for the density. Chem. Phys. Lett. 1998, 287, 233-238.

S7. Gatti, C., The Source Function Descriptor as a Tool to Extract Chemical Information from Theoretical and Experimental Electron Densities. In Electron Density and Chemical Bonding II: Theoretical Charge Density Studies, Stalke, D., Ed. Springer Berlin Heidelberg: Berlin, Heidelberg, 2012; pp 193-285.

S8. Zhang, L.; Ying, F.; Wu, W.; Hiberty, P. C.; Shaik, S. Topology of electron charge density for chemical bonds from valence bond theory: a probe of bonding types. Chem. Eur. J. 2009, 15, 2979-89. 


\section{Gamess-US (2017-R1) and XMVB-3.0 Input Files}

Below we append the Gamess-US input files and XMVB input files for the complexes discussed in the manuscript. Interested readers can obtain the output files from the authors upon request.

\section{$\underline{\text { 1. } \mathrm{H}-\mathrm{NO}_{2}}$}

\section{Gamess-US input}

$\$$ contrl runtyp $=$ energy $\operatorname{scftyp}=\operatorname{rhf}$ maxit $=200$ icharg $=0$ mult $=1$ vbtyp $=x m v b \$$ end $\$$ scf damp=.t. $\operatorname{soscf}=$. f. diis=.t. $\$$ end

\$system mwords $=125$ \$end

\$basis basnam $(1)=\mathrm{N}, \mathrm{O}, \mathrm{O}, \mathrm{H}$, Send

\$data

$\mathrm{NO} 2 \mathrm{H}$ at cc-pVDZ basis set

$\mathrm{C} 1$

$\begin{array}{lcccr}\text { NITROGEN } & 7.0 & 0.000000000 & 0.000000000 & 0.518274000 \\ \text { OXYGEN } & 8.0 & 1.097977000 & 0.000000000 & 1.048619000 \\ \text { OXYGEN } & 8.0 & -1.097977000 & 0.000000000 & 1.048619000 \\ \text { HYDROGEN } & 1.0 & 0.000000000 & 0.000000000 & -0.518274000\end{array}$

Send

\begin{tabular}{llll}
\multicolumn{2}{l}{ \$N } & & \\
$\mathrm{S}$ & 9 & & \\
1 & & $9.046000 \mathrm{E}+03$ & $7.000000 \mathrm{E}-04$ \\
2 & & $1.357000 \mathrm{E}+03$ & $5.389000 \mathrm{E}-03$ \\
3 & & $3.093000 \mathrm{E}+02$ & $2.740600 \mathrm{E}-02$ \\
4 & & $8.773000 \mathrm{E}+01$ & $1.032070 \mathrm{E}-01$ \\
5 & & $2.856000 \mathrm{E}+01$ & $2.787230 \mathrm{E}-01$ \\
6 & & $1.021000 \mathrm{E}+01$ & $4.485400 \mathrm{E}-01$ \\
7 & & $3.838000 \mathrm{E}+00$ & $2.782380 \mathrm{E}-01$ \\
8 & & $7.466000 \mathrm{E}-01$ & $1.544000 \mathrm{E}-02$ \\
9 & & $2.248000 \mathrm{E}-01$ & $-2.864000 \mathrm{E}-03$ \\
$\mathrm{~S}$ & 9 & & \\
1 & & $9.046000 \mathrm{E}+03$ & $-1.530000 \mathrm{E}-04$ \\
2 & & $1.357000 \mathrm{E}+03$ & $-1.208000 \mathrm{E}-03$ \\
3 & & $3.093000 \mathrm{E}+02$ & $-5.992000 \mathrm{E}-03$ \\
4 & & $8.773000 \mathrm{E}+01$ & $-2.454400 \mathrm{E}-02$ \\
5 & & $2.856000 \mathrm{E}+01$ & $-6.745900 \mathrm{E}-02$ \\
6 & & $1.021000 \mathrm{E}+01$ & $-1.580780 \mathrm{E}-01$ \\
7 & & $3.838000 \mathrm{E}+00$ & $-1.218310 \mathrm{E}-01$ \\
8 & & $7.466000 \mathrm{E}-01$ & $5.490030 \mathrm{E}-01$ \\
9 & & $2.248000 \mathrm{E}-01$ & $5.788150 \mathrm{E}-01$ \\
$\mathrm{~S}$ & 1 & & \\
1 & & $2.248000 \mathrm{E}-01$ & $1.000000 \mathrm{E}+00$ \\
$\mathrm{P}$ & 4 & &
\end{tabular}




$\begin{array}{llll}1 & & 1.355000 \mathrm{E}+01 & 3.991900 \mathrm{E}-02 \\ 2 & & 2.917000 \mathrm{E}+00 & 2.171690 \mathrm{E}-01 \\ 3 & & 7.973000 \mathrm{E}-01 & 5.103190 \mathrm{E}-01 \\ 4 & & 2.185000 \mathrm{E}-01 & 4.622140 \mathrm{E}-01 \\ \mathrm{P} & 1 & & \\ 1 & & 2.185000 \mathrm{E}-01 & 1.000000 \mathrm{E}+00 \\ \mathrm{D} & 1 & & \\ 1 & & 8.170000 \mathrm{E}-01 & 1.0000000\end{array}$

\begin{tabular}{|c|c|c|}
\hline \multicolumn{3}{|c|}{ \$END } \\
\hline \multicolumn{3}{|c|}{$\$ O$} \\
\hline \multicolumn{3}{|c|}{59} \\
\hline 1 & $1.172000 \mathrm{E}+04$ & $7.100000 \mathrm{E}-04$ \\
\hline 2 & $1.759000 \mathrm{E}+03$ & $5.470000 \mathrm{E}-03$ \\
\hline 3 & $4.008000 \mathrm{E}+02$ & $2.783700 \mathrm{E}-02$ \\
\hline 4 & $1.137000 \mathrm{E}+02$ & $1.048000 \mathrm{E}-01$ \\
\hline 5 & $3.703000 \mathrm{E}+01$ & $2.830620 \mathrm{E}-01$ \\
\hline 6 & $1.327000 \mathrm{E}+01$ & 4.487190E-01 \\
\hline 7 & $5.025000 \mathrm{E}+00$ & $2.709520 \mathrm{E}-01$ \\
\hline 8 & $1.013000 \mathrm{E}+00$ & $1.545800 \mathrm{E}-02$ \\
\hline 9 & $3.023000 \mathrm{E}-01$ & $-2.585000 \mathrm{E}-03$ \\
\hline \multicolumn{3}{|r|}{2.00000000} \\
\hline 1 & $1.172000 \mathrm{E}+04$ & $-1.600000 \mathrm{E}-04$ \\
\hline 2 & $1.759000 \mathrm{E}+03$ & $-1.263000 \mathrm{E}-03$ \\
\hline 3 & $4.008000 \mathrm{E}+02$ & $-6.267000 \mathrm{E}-03$ \\
\hline 4 & $1.137000 \mathrm{E}+02$ & $-2.571600 \mathrm{E}-02$ \\
\hline 5 & $3.703000 \mathrm{E}+01$ & $-7.092400 \mathrm{E}-02$ \\
\hline 6 & $1.327000 \mathrm{E}+01$ & $-1.654110 \mathrm{E}-01$ \\
\hline 7 & $5.025000 \mathrm{E}+00$ & $-1.169550 \mathrm{E}-01$ \\
\hline 8 & $1.013000 \mathrm{E}+00$ & $5.573680 \mathrm{E}-01$ \\
\hline 9 & $3.023000 \mathrm{E}-01$ & $5.727590 \mathrm{E}-01$ \\
\hline \multicolumn{3}{|r|}{ 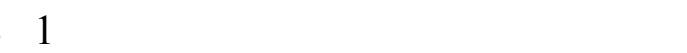 } \\
\hline 1 & $3.023000 \mathrm{E}-01$ & $1.000000 \mathrm{E}+00$ \\
\hline \multicolumn{3}{|r|}{1.0000000 .00} \\
\hline 1 & $1.770000 \mathrm{E}+01$ & 4.301800E-02 \\
\hline 2 & $3.854000 \mathrm{E}+00$ & $2.289130 \mathrm{E}-01$ \\
\hline 3 & $1.046000 \mathrm{E}+00$ & $5.087280 \mathrm{E}-01$ \\
\hline 4 & $2.753000 \mathrm{E}-01$ & $4.605310 \mathrm{E}-01$ \\
\hline $\mathrm{P}$ & & \\
\hline 1 & $2.753000 \mathrm{E}-01$ & $1.000000 \mathrm{E}+00$ \\
\hline $\mathrm{D}$ & & \\
\hline 1 & $1.185000 \mathrm{E}+00$ & 1.0000000 \\
\hline
\end{tabular}

\begin{tabular}{|c|c|c|}
\hline \\
\hline \\
\hline \multicolumn{3}{|c|}{$\begin{array}{l}\text { \$END } \\
\text { \$H } \\
\text { S } 4\end{array}$} \\
\hline \multicolumn{3}{|c|}{$1.301000 \mathrm{E}+01$} \\
\hline & \multirow{2}{*}{$\begin{array}{l}1.962000 \mathrm{E}+00 \\
4.446000 \mathrm{E}-01\end{array}$} & $1.379770 \mathrm{E}-01$ \\
\hline & & 4.781480E-01 \\
\hline 4 & $1.220000 \mathrm{E}-01$ & $5.012400 \mathrm{E}-01$ \\
\hline
\end{tabular}




\begin{tabular}{llll}
$\mathrm{S}$ & 1 & & \\
1 & & $1.220000 \mathrm{E}-01$ & $1.000000 \mathrm{E}+00$ \\
$\mathrm{P}$ & 1 & & \\
1 & & $7.270000 \mathrm{E}-01$ & 1.0000000 \\
& & \\
\multicolumn{3}{l}{ SEND } &
\end{tabular}

\section{$\underline{\text { XMVB input }}$}

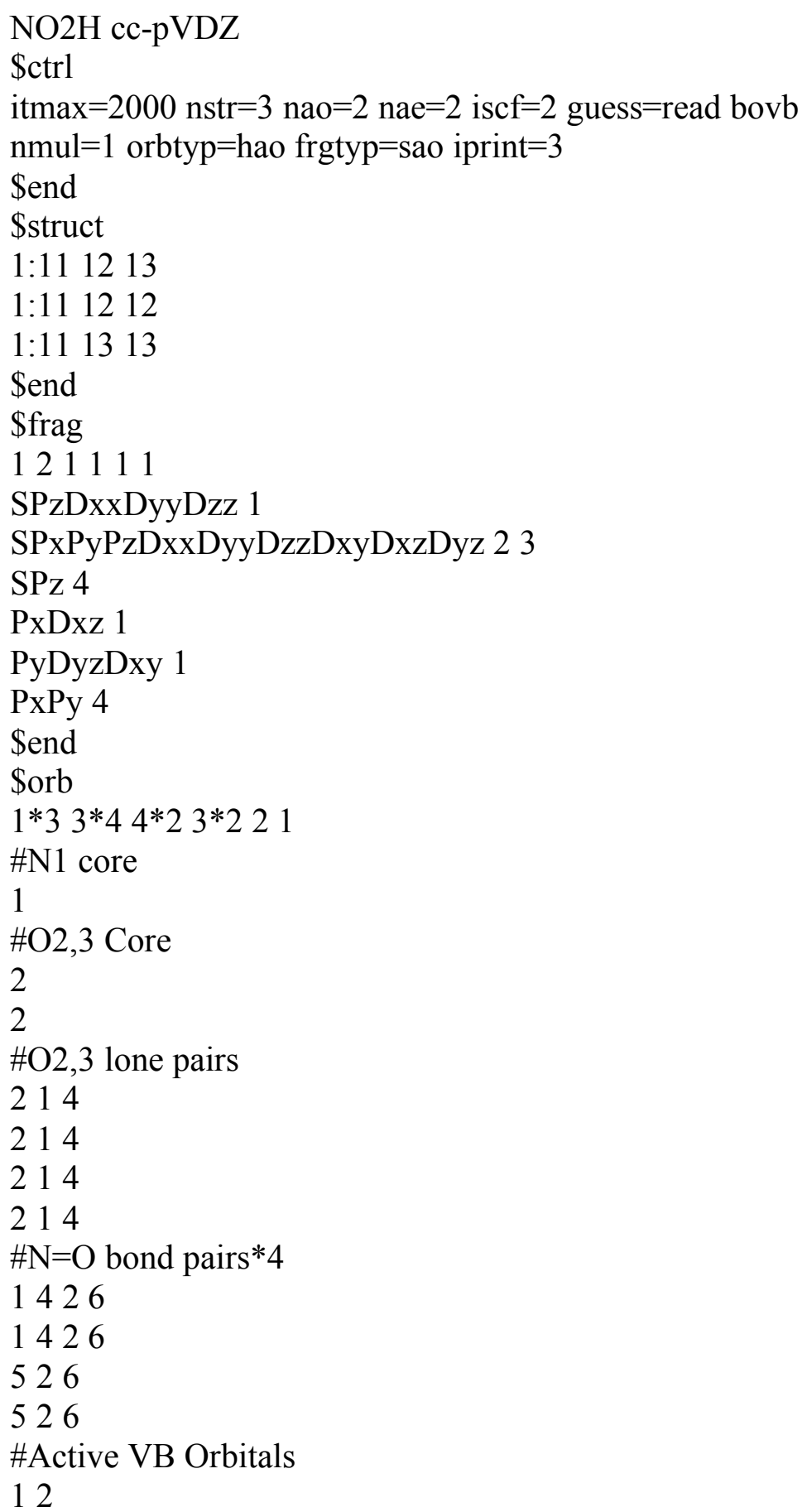




\section{$\underline{\text { 2. } \mathrm{H}_{3} \mathrm{C}-\mathrm{NO}_{2}}$}

\section{Gamess-US input}

$\$$ contrl runtyp=energy scftyp $=\mathrm{rhf}$ maxit $=200$ icharg $=0$ mult $=1$ vbtyp=xmvb $\$$ end $\$ \mathrm{scf}$ damp=.t. $\operatorname{soscf}=$.f. diis=.t. $\$$ end

\$system mwords $=125$ Send

\$basis basnam $(1)=\mathrm{N}, \mathrm{O}, \mathrm{O}, \mathrm{C}, \mathrm{H}, \mathrm{H}, \mathrm{H}$, Send

\$data

NO2Me at cc-pVDZ basis set

$\mathrm{C} 1$

$\begin{array}{lrrrr}\text { NITROGEN } & 7.0 & 0.000000000 & 0.000000000 & 0.746355995 \\ \text { OXYGEN } & 8.0 & 1.090036626 & 0.000000000 & 1.303237895 \\ \text { OXYGEN } & 8.0 & -1.089707183 & 0.028261487 & 1.303166905 \\ \text { CARBON } & 6.0 & 0.000000000 & 0.000000000 & -0.746355995 \\ \text { HYDROGEN } & 1.0 & 0.012389047 & 1.039531889 & -1.059451750 \\ \text { HYDROGEN } & 1.0 & 0.895971730 & -0.506548415 & -1.080732930 \\ \text { HYDROGEN } & 1.0 & -0.907876658 & -0.484934328 & -1.080686256\end{array}$

Send

\begin{tabular}{llll}
\multicolumn{2}{c}{$\$ \mathrm{H}$} & & \\
$\mathrm{S}$ & 4 & & \\
1 & & $1.301000 \mathrm{E}+01$ & $1.968500 \mathrm{E}-02$ \\
2 & & $1.962000 \mathrm{E}+00$ & $1.379770 \mathrm{E}-01$ \\
3 & & $4.446000 \mathrm{E}-01$ & $4.781480 \mathrm{E}-01$ \\
4 & & $1.220000 \mathrm{E}-01$ & $5.012400 \mathrm{E}-01$ \\
$\mathrm{~S}$ & 1 & & \\
1 & & $1.220000 \mathrm{E}-01$ & $1.000000 \mathrm{E}+00$ \\
$\mathrm{P}$ & 1 & & \\
1 & & $7.270000 \mathrm{E}-01$ & 1.0000000 \\
& & \\
\$END & & \\
\$C & & \\
$\mathrm{S}$ & 9 & & \\
1 & & $6.665000 \mathrm{E}+03$ & $6.920000 \mathrm{E}-04$ \\
2 & & $1.000000 \mathrm{E}+03$ & $5.329000 \mathrm{E}-03$ \\
3 & & $2.280000 \mathrm{E}+02$ & $2.707700 \mathrm{E}-02$ \\
4 & & $6.471000 \mathrm{E}+01$ & $1.017180 \mathrm{E}-01$ \\
5 & & $2.106000 \mathrm{E}+01$ & $2.747400 \mathrm{E}-01$ \\
6 & & $7.495000 \mathrm{E}+00$ & $4.485640 \mathrm{E}-01$ \\
7 & & $2.797000 \mathrm{E}+00$ & $2.850740 \mathrm{E}-01$ \\
8 & & $5.215000 \mathrm{E}-01$ & $1.520400 \mathrm{E}-02$ \\
9 & & $1.596000 \mathrm{E}-01$ & $-3.191000 \mathrm{E}-03$ \\
$\mathrm{~S}$ & 9 & &
\end{tabular}




$\begin{array}{llll}1 & & 6.665000 \mathrm{E}+03 & -1.460000 \mathrm{E}-04 \\ 2 & & 1.000000 \mathrm{E}+03 & -1.154000 \mathrm{E}-03 \\ 3 & & 2.280000 \mathrm{E}+02 & -5.725000 \mathrm{E}-03 \\ 4 & & 6.471000 \mathrm{E}+01 & -2.331200 \mathrm{E}-02 \\ 5 & & 2.106000 \mathrm{E}+01 & -6.395500 \mathrm{E}-02 \\ 6 & & 7.495000 \mathrm{E}+00 & -1.499810 \mathrm{E}-01 \\ 7 & & 2.797000 \mathrm{E}+00 & -1.272620 \mathrm{E}-01 \\ 8 & & 5.215000 \mathrm{E}-01 & 5.445290 \mathrm{E}-01 \\ 9 & & 1.596000 \mathrm{E}-01 & 5.804960 \mathrm{E}-01 \\ \mathrm{~S} & 1 & & \\ 1 & & 1.596000 \mathrm{E}-01 & 1.000000 \mathrm{E}+00 \\ \mathrm{P} & 4 & & \\ 1 & & 9.439000 \mathrm{E}+00 & 3.810900 \mathrm{E}-02 \\ 2 & & 2.002000 \mathrm{E}+00 & 2.094800 \mathrm{E}-01 \\ 3 & & 5.456000 \mathrm{E}-01 & 5.085570 \mathrm{E}-01 \\ 4 & & 1.517000 \mathrm{E}-01 & 4.688420 \mathrm{E}-01 \\ \mathrm{P} & 1 & & \\ 1 & & 1.517000 \mathrm{E}-01 & 1.000000 \mathrm{E}+00 \\ \mathrm{D} & 1 & & \\ 1 & & 5.500000 \mathrm{E}-01 & 1.0000000\end{array}$

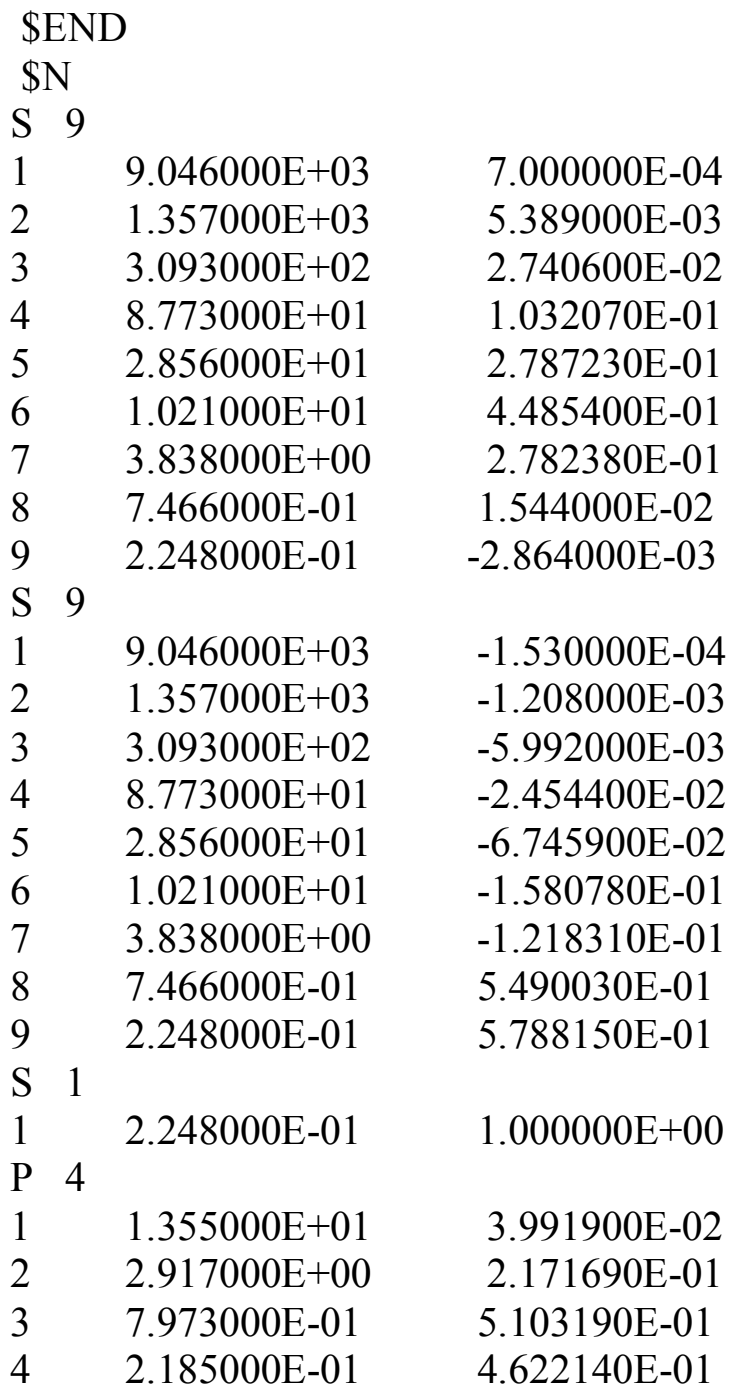




\begin{tabular}{|c|c|c|}
\hline \multicolumn{3}{|c|}{ P 1} \\
\hline 1 & $2.185000 \mathrm{E}-01$ & $1.000000 \mathrm{E}+00$ \\
\hline \multicolumn{3}{|c|}{ D 1} \\
\hline 1 & $8.170000 \mathrm{E}-01$ & 1.0000000 \\
\hline \multicolumn{3}{|c|}{ \$END } \\
\hline \multicolumn{3}{|c|}{$\$ O$} \\
\hline \multicolumn{3}{|c|}{ S 9} \\
\hline 1 & $1.172000 \mathrm{E}+04$ & 7.100000E-04 \\
\hline 2 & $1.759000 \mathrm{E}+03$ & $5.470000 \mathrm{E}-03$ \\
\hline 3 & $4.008000 \mathrm{E}+02$ & $2.783700 \mathrm{E}-02$ \\
\hline 4 & $1.137000 \mathrm{E}+02$ & $1.048000 \mathrm{E}-01$ \\
\hline 5 & $3.703000 \mathrm{E}+01$ & $2.830620 \mathrm{E}-01$ \\
\hline 6 & $1.327000 \mathrm{E}+01$ & 4.487190E-01 \\
\hline 7 & $5.025000 \mathrm{E}+00$ & $2.709520 \mathrm{E}-01$ \\
\hline 8 & $1.013000 \mathrm{E}+00$ & $1.545800 \mathrm{E}-02$ \\
\hline 9 & $3.023000 \mathrm{E}-01$ & $-2.585000 \mathrm{E}-03$ \\
\hline \multicolumn{3}{|r|}{$2.000000 \mathrm{D}=0$} \\
\hline 1 & $1.172000 \mathrm{E}+04$ & $-1.600000 \mathrm{E}-04$ \\
\hline 2 & $1.759000 \mathrm{E}+03$ & $-1.263000 \mathrm{E}-03$ \\
\hline 3 & $4.008000 \mathrm{E}+02$ & $-6.267000 \mathrm{E}-03$ \\
\hline 4 & $1.137000 \mathrm{E}+02$ & $-2.571600 \mathrm{E}-02$ \\
\hline 5 & $3.703000 \mathrm{E}+01$ & $-7.092400 \mathrm{E}-02$ \\
\hline 6 & $1.327000 \mathrm{E}+01$ & $-1.654110 \mathrm{E}-01$ \\
\hline 7 & $5.025000 \mathrm{E}+00$ & $-1.169550 \mathrm{E}-01$ \\
\hline 8 & $1.013000 \mathrm{E}+00$ & $5.573680 \mathrm{E}-01$ \\
\hline 9 & $3.023000 \mathrm{E}-01$ & $5.727590 \mathrm{E}-01$ \\
\hline \multicolumn{3}{|c|}{$\begin{array}{ll}S & 1\end{array}$} \\
\hline 1 & $3.023000 \mathrm{E}-01$ & $1.000000 \mathrm{E}+00$ \\
\hline \multicolumn{3}{|c|}{$\mathrm{P} 4$} \\
\hline 1 & $1.770000 \mathrm{E}+01$ & 4.301800E-02 \\
\hline 2 & $3.854000 \mathrm{E}+00$ & $2.289130 \mathrm{E}-01$ \\
\hline 3 & $1.046000 \mathrm{E}+00$ & $5.087280 \mathrm{E}-01$ \\
\hline 4 & $2.753000 \mathrm{E}-01$ & $4.605310 \mathrm{E}-01$ \\
\hline \multicolumn{3}{|c|}{$\mathrm{P} \quad 1$} \\
\hline 1 & $2.753000 \mathrm{E}-01$ & $1.000000 \mathrm{E}+00$ \\
\hline \multicolumn{3}{|c|}{ D 1} \\
\hline 1 & $1.185000 \mathrm{E}+00$ & 1.0000000 \\
\hline
\end{tabular}

\section{$\underline{\text { XMVB input }}$}

NO2Me cc-pVDZ

\$ctrl

itmax $=2000$ nstr $=3$ nao $=2$ nae $=2$ iscf $=2$ guess $=$ read bovb $\mathrm{nmul}=1$ orbtyp $=$ hao frgtyp $=$ sao iprint $=3$ 
Send

\$struct

$1: 151617$

$1: 151616$

$1: 151717$

Send

\$frag

12131111

SPzDxxDyyDzz 1

SPxPyPzDxxDyyDzzDxzDyzDxy 23

SPzDxxDyyDzz 4

SPxPyPz 567

PxDxz 1

PxDxz 4

PyDyzDxy 1

PyDyzDxy 4

Send

\$orb

$1 * 42543 * 45 * 24 * 222$

\#N core

1

\#O core*2

2

2

\#C core

3

\#C-H bonds*3

43

46512

4872

\#O lone pairs

215

215

251

251

$\# \mathrm{~N}=\mathrm{O}$ bond pairs*4

12564

12564

7284

7284

\#Active VB Orbitals

12

34

Send 


\section{$\underline{\text { 3. }\left(\mathrm{H}_{3} \mathrm{C}\right)_{2} \mathrm{~N}-\mathrm{NO}_{2}}$}

\section{Gamess-US input}

$\$$ contrl runtyp=energy scftyp $=$ rhf maxit $=200$ icharg $=0$ mult $=1$ vbtyp=xmvb \$end $\$ \mathrm{scf}$ damp=.t. $\operatorname{soscf}=$.f. diis=.t. $\$$ end

\$system mwords $=125$ \$end

\$basis basnam $(1)=\mathrm{N}, \mathrm{O}, \mathrm{O}, \mathrm{N}, \mathrm{C}, \mathrm{H}, \mathrm{H}, \mathrm{H}, \mathrm{C}, \mathrm{H}, \mathrm{H}, \mathrm{H}$, Send

\$data

NO2-NMe2 at cc-pVDZ basis set

$\mathrm{C} 1$

$\begin{array}{lcccc}\text { NITROGEN } & 7.0 & 0.000000000 & 0.000000000 & 0.691596249 \\ \text { OXYGEN } & 8.0 & 1.093520361 & 0.000000000 & 1.248665999 \\ \text { OXYGEN } & 8.0 & -1.092870948 & 0.037681662 & 1.248665841 \\ \text { NITROGEN } & 7.0 & 0.000000000 & 0.000000000 & -0.691596249 \\ \text { CARBON } & 6.0 & -1.260758915 & -0.388803887 & -1.304315502 \\ \text { HYDROGEN } & 1.0 & -2.058221708 & 0.244543802 & -0.936707529 \\ \text { HYDROGEN } & 1.0 & -1.152399359 & -0.249091721 & -2.375269765 \\ \text { HYDROGEN } & 1.0 & -1.512579371 & -1.431367429 & -1.096839321 \\ \text { CARBON } & 6.0 & 1.246612746 & -0.432016396 & -1.304315502 \\ \text { HYDROGEN } & 1.0 & 1.143131743 & -0.288654319 & -2.375269607 \\ \text { HYDROGEN } & 1.0 & 2.065425921 & 0.173474946 & -0.936708358 \\ \text { HYDROGEN } & 1.0 & 1.462358858 & -1.482638068 & -1.096839321\end{array}$

Send

\begin{tabular}{llll}
\multicolumn{2}{l}{ \$H } & & \\
S & 4 & & \\
1 & & $1.301000 \mathrm{E}+01$ & $1.968500 \mathrm{E}-02$ \\
2 & & $1.962000 \mathrm{E}+00$ & $1.379770 \mathrm{E}-01$ \\
3 & & $4.446000 \mathrm{E}-01$ & $4.781480 \mathrm{E}-01$ \\
4 & & $1.220000 \mathrm{E}-01$ & $5.012400 \mathrm{E}-01$ \\
$\mathrm{~S}$ & 1 & & \\
1 & & $1.220000 \mathrm{E}-01$ & $1.000000 \mathrm{E}+00$ \\
$\mathrm{P}$ & 1 & & \\
1 & & $7.270000 \mathrm{E}-01$ & 1.0000000 \\
& & \\
\$END & & \\
\$C & & \\
S & 9 & & \\
1 & & $6.665000 \mathrm{E}+03$ & $6.920000 \mathrm{E}-04$ \\
2 & & $1.000000 \mathrm{E}+03$ & $5.329000 \mathrm{E}-03$ \\
3 & & $2.280000 \mathrm{E}+02$ & $2.707700 \mathrm{E}-02$ \\
4 & & $6.471000 \mathrm{E}+01$ & $1.017180 \mathrm{E}-01$ \\
5 & & $2.106000 \mathrm{E}+01$ & $2.747400 \mathrm{E}-01$ \\
6 & & $7.495000 \mathrm{E}+00$ & $4.485640 \mathrm{E}-01$ \\
7 & & $2.797000 \mathrm{E}+00$ & $2.850740 \mathrm{E}-01$ \\
8 & & $5.215000 \mathrm{E}-01$ & $1.520400 \mathrm{E}-02$ \\
9 & & $1.596000 \mathrm{E}-01$ & $-3.191000 \mathrm{E}-03$ \\
$\mathrm{~S}$ & 9 & & \\
1 & & $6.665000 \mathrm{E}+03$ & $-1.460000 \mathrm{E}-04$
\end{tabular}




\begin{tabular}{|c|c|c|}
\hline 2 & $1.000000 \mathrm{E}+03$ & $-1.154000 \mathrm{E}-03$ \\
\hline 3 & $2.280000 \mathrm{E}+02$ & $-5.725000 \mathrm{E}-03$ \\
\hline 4 & $6.471000 \mathrm{E}+01$ & $-2.331200 \mathrm{E}-02$ \\
\hline 5 & $2.106000 \mathrm{E}+01$ & $-6.395500 \mathrm{E}-02$ \\
\hline 6 & $7.495000 \mathrm{E}+00$ & $-1.499810 \mathrm{E}-01$ \\
\hline 7 & $2.797000 \mathrm{E}+00$ & $-1.272620 \mathrm{E}-01$ \\
\hline 8 & $5.215000 \mathrm{E}-01$ & $5.445290 \mathrm{E}-01$ \\
\hline 9 & $1.596000 \mathrm{E}-01$ & $5.804960 \mathrm{E}-01$ \\
\hline & & \\
\hline 1 & $1.596000 \mathrm{E}-01$ & $1.000000 \mathrm{E}+00$ \\
\hline $\mathrm{P}$ & & \\
\hline 1 & $9.439000 \mathrm{E}+00$ & $3.810900 \mathrm{E}-02$ \\
\hline 2 & $2.002000 \mathrm{E}+00$ & $2.094800 \mathrm{E}-01$ \\
\hline 3 & $5.456000 \mathrm{E}-01$ & $5.085570 \mathrm{E}-01$ \\
\hline 4 & $1.517000 \mathrm{E}-01$ & $4.688420 \mathrm{E}-01$ \\
\hline $\mathrm{P}$ & & \\
\hline 1 & $1.517000 \mathrm{E}-01$ & $1.000000 \mathrm{E}+00$ \\
\hline D & & \\
\hline 1 & $5.500000 \mathrm{E}-01$ & 1.0000000 \\
\hline & & \\
\hline$\$ 1$ & & \\
\hline S & & \\
\hline 1 & $9.046000 \mathrm{E}+03$ & $7.000000 \mathrm{E}-04$ \\
\hline 2 & $1.357000 \mathrm{E}+03$ & $5.389000 \mathrm{E}-03$ \\
\hline 3 & $3.093000 \mathrm{E}+02$ & $2.740600 \mathrm{E}-02$ \\
\hline 4 & $8.773000 \mathrm{E}+01$ & $1.032070 \mathrm{E}-01$ \\
\hline 5 & $2.856000 \mathrm{E}+01$ & $2.787230 \mathrm{E}-01$ \\
\hline 6 & $1.021000 \mathrm{E}+01$ & $4.485400 \mathrm{E}-01$ \\
\hline 7 & $3.838000 \mathrm{E}+00$ & $2.782380 \mathrm{E}-01$ \\
\hline 8 & $7.466000 \mathrm{E}-01$ & $1.544000 \mathrm{E}-02$ \\
\hline 9 & $2.248000 \mathrm{E}-01$ & $-2.864000 \mathrm{E}-03$ \\
\hline S & & \\
\hline 1 & $9.046000 \mathrm{E}+03$ & $-1.530000 \mathrm{E}-04$ \\
\hline 2 & $1.357000 \mathrm{E}+03$ & $-1.208000 \mathrm{E}-03$ \\
\hline 3 & $3.093000 \mathrm{E}+02$ & $-5.992000 \mathrm{E}-03$ \\
\hline 4 & $8.773000 \mathrm{E}+01$ & $-2.454400 \mathrm{E}-02$ \\
\hline 5 & $2.856000 \mathrm{E}+01$ & $-6.745900 \mathrm{E}-02$ \\
\hline 6 & $1.021000 \mathrm{E}+01$ & $-1.580780 \mathrm{E}-01$ \\
\hline 7 & $3.838000 \mathrm{E}+00$ & $-1.218310 \mathrm{E}-01$ \\
\hline 8 & $7.466000 \mathrm{E}-01$ & $5.490030 \mathrm{E}-01$ \\
\hline 9 & $2.248000 \mathrm{E}-01$ & $5.788150 \mathrm{E}-01$ \\
\hline $\mathrm{S}$ & & \\
\hline 1 & $2.248000 \mathrm{E}-01$ & $1.000000 \mathrm{E}+00$ \\
\hline $\mathrm{P}$ & & \\
\hline 1 & $1.355000 \mathrm{E}+01$ & $3.991900 \mathrm{E}-02$ \\
\hline 2 & $2.917000 \mathrm{E}+00$ & $2.171690 \mathrm{E}-01$ \\
\hline 3 & $7.973000 \mathrm{E}-01$ & $5.103190 \mathrm{E}-01$ \\
\hline 4 & $2.185000 \mathrm{E}-01$ & $4.622140 \mathrm{E}-01$ \\
\hline $\mathrm{P}$ & & \\
\hline
\end{tabular}




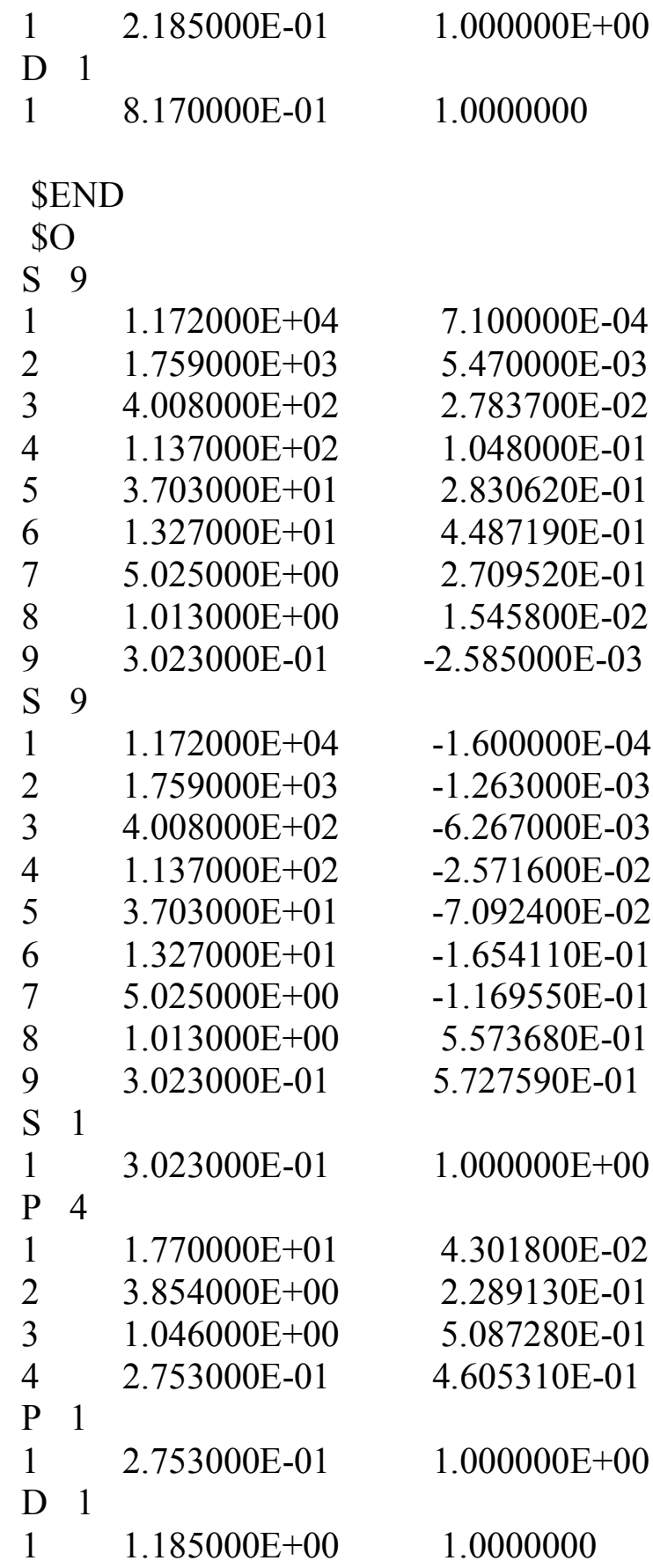

SEND

\section{$\underline{\text { XMVB input }}$}

NO2-NMe2 cc-pVDZ

\$ctrl

itmax $=2000 \mathrm{nstr}=3$ nao $=2$ nae $=2$ iscf $=2$ guess $=$ read bovb $\mathrm{nmul}=1$ orbtyp $=$ hao frgtyp $=$ sao iprint $=3$ 
Send

\$struct

1:23 2425

$1: 232424$

$1: 232525$

Send

\$frag

12113131211112111

SPzDxxDyyDzz 1

SPzDxxDyyDzz 23

SPzDxxDyyDzz 4

SPzDxxDyyDzz 5

SPxPyPz 678

SPzDxxDyyDzz 9

SPxPyPz 101112

PxDxz 1

PxDxz 23

PxDxz 4

PxDxz 5

PxDxz 9

PyDyzDxy 1

PyDyzDxy 23

PyDyzDxy 4

PyDyzDxy 5

PyDyzDxy 9

Send

\$orb

$1 * 64 * 23 * 28 * 28 * 64 * 4825$

\#N1 core

1

\#O2,3 core*2

2

2

\#N4 core

3

\#C5 core

4

\#C9 core

6

\#N1=O2,3 bond pairs $* 4$

1298

1298

131415

131415

\#N4-C5 bond

31041156127

\#N4-C9 bond

31061274115

\#C5-H6,7,8 bond

541116761217 
541116761217

541116761217

\#C9-H10,11,12 bond

761217541116

761217541116

761217541116

\#O2,3 lone pairs

2918

2918

9218

9218

\#N4-lone pair

1531314161757

\#Active VB Orbitals

12

34567

Send

\section{4. $\left(\mathrm{H}_{3} \mathrm{C}\right) \mathrm{O}-\mathrm{NO}_{2}$}

\section{Gamess-US input}

$\$$ contrl runtyp $=$ energy $s c f t y p=\operatorname{rhf}$ maxit $=200$ icharg $=0$ mult $=1$ vbtyp $=x m v b \$$ end $\$$ scf damp=.t. $\operatorname{soscf}=$.f. diis=.t. $\$$ end

\$system mwords $=125$ \$end

\$basis basnam $(1)=\mathrm{N}, \mathrm{O}, \mathrm{O}, \mathrm{O}, \mathrm{C}, \mathrm{H}, \mathrm{H}, \mathrm{H}$, Send

\$data

NO2-OMe at cc-pVDZ basis set

$\mathrm{C} 1$

$\begin{array}{lrrrr}\text { NITROGEN } & 7.0 & 0.000000000 & 0.000000000 & 0.709101263 \\ \text { OXYGEN } & 8.0 & 1.075731860 & 0.000000000 & 1.262007676 \\ \text { OXYGEN } & 8.0 & -1.108206005 & 0.000000455 & 1.171424985 \\ \text { OXYGEN } & 8.0 & 0.000000000 & 0.000000000 & -0.709101263 \\ \text { CARBON } & 6.0 & 1.322866241 & -0.000004742 & -1.264461995 \\ \text { HYDROGEN } & 1.0 & 1.870237025 & -0.889918720 & -0.967643980 \\ \text { HYDROGEN } & 1.0 & 1.145611013 & -0.000009123 & -2.334741806 \\ \text { HYDROGEN } & 1.0 & 1.870240368 & 0.889910280 & -0.967651158\end{array}$

\$end

$\$ \mathrm{H}$

S 4

$1 \quad 1.301000 \mathrm{E}+01 \quad 1.968500 \mathrm{E}-02$

$2 \quad 1.962000 \mathrm{E}+00 \quad 1.379770 \mathrm{E}-01$

$3 \quad 4.446000 \mathrm{E}-01 \quad 4.781480 \mathrm{E}-01$

$4 \quad 1.220000 \mathrm{E}-01 \quad 5.012400 \mathrm{E}-01$

S 1

$1 \quad 1.220000 \mathrm{E}-01 \quad 1.000000 \mathrm{E}+00$ 
P 1

$\begin{array}{lll}P & 7.270000 \mathrm{E}-01 & 1.0000000\end{array}$

\begin{tabular}{llll}
\multicolumn{3}{l}{ \$END } & \\
\$C & & \\
S & & & \\
1 & & $6.665000 \mathrm{E}+03$ & $6.920000 \mathrm{E}-04$ \\
2 & & $1.000000 \mathrm{E}+03$ & $5.329000 \mathrm{E}-03$ \\
3 & & $2.280000 \mathrm{E}+02$ & $2.707700 \mathrm{E}-02$ \\
4 & & $6.471000 \mathrm{E}+01$ & $1.017180 \mathrm{E}-01$ \\
5 & & $2.106000 \mathrm{E}+01$ & $2.747400 \mathrm{E}-01$ \\
6 & & $7.495000 \mathrm{E}+00$ & $4.485640 \mathrm{E}-01$ \\
7 & & $2.797000 \mathrm{E}+00$ & $2.850740 \mathrm{E}-01$ \\
8 & & $5.215000 \mathrm{E}-01$ & $1.520400 \mathrm{E}-02$ \\
9 & & $1.596000 \mathrm{E}-01$ & $-3.191000 \mathrm{E}-03$ \\
$\mathrm{~S}$ & 9 & & \\
1 & & $6.665000 \mathrm{E}+03$ & $-1.460000 \mathrm{E}-04$ \\
2 & & $1.000000 \mathrm{E}+03$ & $-1.154000 \mathrm{E}-03$ \\
3 & & $2.280000 \mathrm{E}+02$ & $-5.725000 \mathrm{E}-03$ \\
4 & & $6.471000 \mathrm{E}+01$ & $-2.331200 \mathrm{E}-02$ \\
5 & & $2.106000 \mathrm{E}+01$ & $-6.395500 \mathrm{E}-02$ \\
6 & & $7.495000 \mathrm{E}+00$ & $-1.499810 \mathrm{E}-01$ \\
7 & & $2.797000 \mathrm{E}+00$ & $-1.272620 \mathrm{E}-01$ \\
8 & & $5.215000 \mathrm{E}-01$ & $5.445290 \mathrm{E}-01$ \\
9 & & $1.596000 \mathrm{E}-01$ & $5.804960 \mathrm{E}-01$ \\
$\mathrm{~S}$ & 1 & & \\
1 & & $1.596000 \mathrm{E}-01$ & $1.000000 \mathrm{E}+00$ \\
$\mathrm{P}$ & 4 & & \\
1 & & $9.439000 \mathrm{E}+00$ & $3.810900 \mathrm{E}-02$ \\
2 & & $2.002000 \mathrm{E}+00$ & $2.094800 \mathrm{E}-01$ \\
3 & & $5.456000 \mathrm{E}-01$ & $5.085570 \mathrm{E}-01$ \\
4 & & $1.517000 \mathrm{E}-01$ & $4.688420 \mathrm{E}-01$ \\
$\mathrm{P}$ & 1 & & \\
1 & & $1.517000 \mathrm{E}-01$ & $1.000000 \mathrm{E}+00$ \\
$\mathrm{D}$ & 1 & & \\
1 & & $5.500000 \mathrm{E}-01$ & 1.0000000 \\
& &
\end{tabular}

\begin{tabular}{|c|c|c|}
\hline \multicolumn{3}{|c|}{ \$END } \\
\hline \multicolumn{3}{|c|}{$\$ \mathrm{~N}$} \\
\hline S & & \\
\hline 1 & $9.046000 \mathrm{E}+03$ & $7.000000 \mathrm{E}-04$ \\
\hline 2 & $1.357000 \mathrm{E}+03$ & $5.389000 \mathrm{E}-03$ \\
\hline 3 & $3.093000 \mathrm{E}+02$ & $2.740600 \mathrm{E}-02$ \\
\hline 4 & $8.773000 \mathrm{E}+01$ & $1.032070 \mathrm{E}-01$ \\
\hline 5 & $2.856000 \mathrm{E}+01$ & $2.787230 \mathrm{E}-01$ \\
\hline 6 & $1.021000 \mathrm{E}+01$ & 4.485400E-01 \\
\hline 7 & $3.838000 \mathrm{E}+00$ & $2.782380 \mathrm{E}-01$ \\
\hline 8 & $7.466000 \mathrm{E}-01$ & $1.544000 \mathrm{E}-02$ \\
\hline 9 & $2.248000 \mathrm{E}-01$ & $-2.864000 \mathrm{E}-03$ \\
\hline
\end{tabular}




$\begin{array}{llll}1 & & 9.046000 \mathrm{E}+03 & -1.530000 \mathrm{E}-04 \\ 2 & & 1.357000 \mathrm{E}+03 & -1.208000 \mathrm{E}-03 \\ 3 & & 3.093000 \mathrm{E}+02 & -5.992000 \mathrm{E}-03 \\ 4 & & 8.773000 \mathrm{E}+01 & -2.454400 \mathrm{E}-02 \\ 5 & & 2.856000 \mathrm{E}+01 & -6.745900 \mathrm{E}-02 \\ 6 & & 1.021000 \mathrm{E}+01 & -1.580780 \mathrm{E}-01 \\ 7 & & 3.838000 \mathrm{E}+00 & -1.218310 \mathrm{E}-01 \\ 8 & & 7.466000 \mathrm{E}-01 & 5.490030 \mathrm{E}-01 \\ 9 & & 2.248000 \mathrm{E}-01 & 5.788150 \mathrm{E}-01 \\ \mathrm{~S} & 1 & & \\ 1 & & 2.248000 \mathrm{E}-01 & 1.000000 \mathrm{E}+00 \\ \mathrm{P} & 4 & & \\ 1 & & 1.355000 \mathrm{E}+01 & 3.991900 \mathrm{E}-02 \\ 2 & & 2.917000 \mathrm{E}+00 & 2.171690 \mathrm{E}-01 \\ 3 & & 7.973000 \mathrm{E}-01 & 5.103190 \mathrm{E}-01 \\ 4 & & 2.185000 \mathrm{E}-01 & 4.622140 \mathrm{E}-01 \\ \mathrm{P} & 1 & & \\ 1 & & 2.185000 \mathrm{E}-01 & 1.000000 \mathrm{E}+00 \\ \mathrm{D} & 1 & & \\ 1 & & 8.170000 \mathrm{E}-01 & 1.0000000\end{array}$

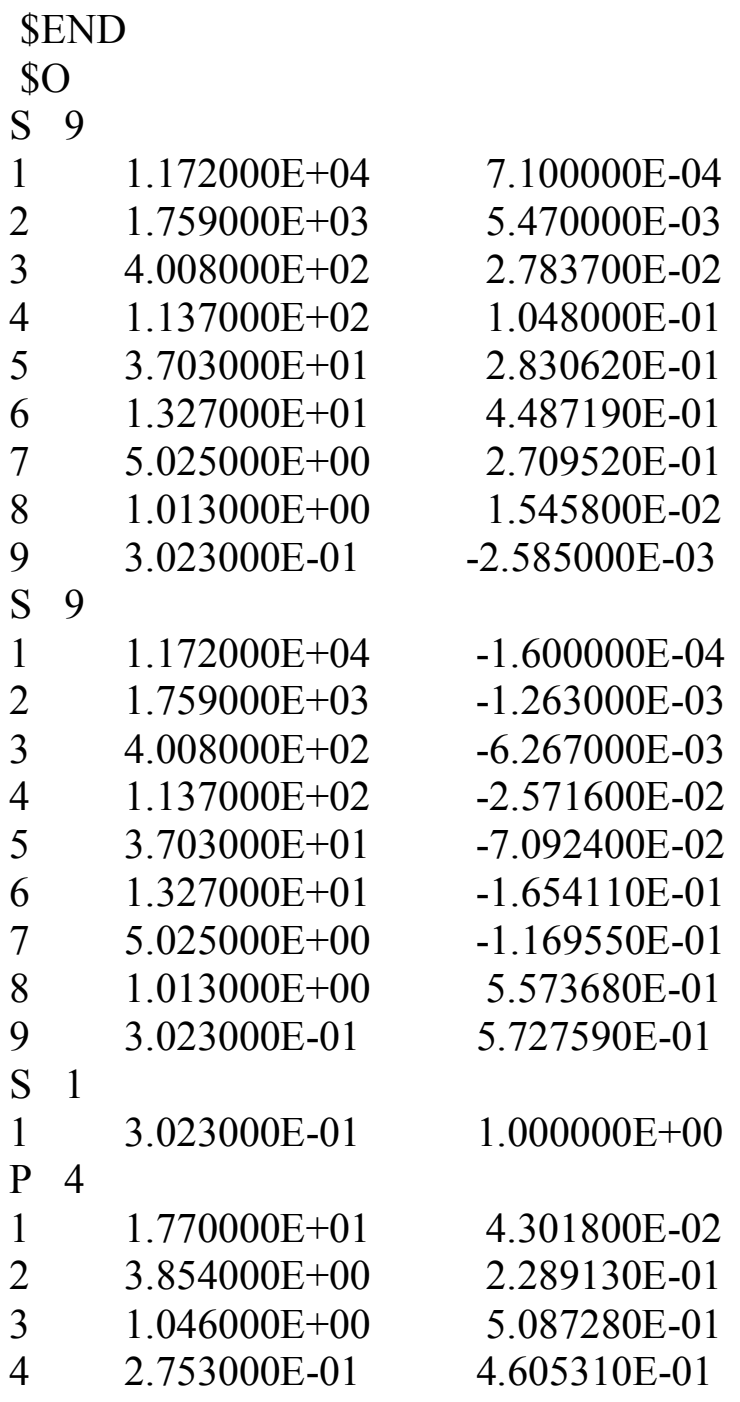


P 1

$1 \quad 2.753000 \mathrm{E}-01 \quad 1.000000 \mathrm{E}+00$

D 1

$1 \quad 1.185000 \mathrm{E}+00 \quad 1.0000000$

\$END

\section{$\underline{\text { XMVB input }}$}

NO2-OMe cc-pVDZ

\$ctrl

itmax $=2000 \mathrm{nstr}=3$ nao $=2$ nae $=2$ isc $=2$ guess $=$ read bovb

nmul $=1$ orbtyp $=$ hao frgtyp $=$ sao iprint $=3$

\$end

\$struct

$1: 192021$

$1: 192020$

$1: 192121$

\$end

$\$$ frag

121131111

SPzDxxDyyDzz 1

SPxPyPzDxxDyyDzzDxyDxzDy 23

SPzDxxDyyDzz 4

SPxPyPzDxxDyyDzzDxyDxzDyz 5

SPxPyPz 678

PxDxz 1

PxDxz 4

PyDyzDxy 1

PyDyzDxy 4

Send

\$orb

$1 * 53 * 25 * 242 * 3453 * 422$

\#N1 core

1

\#O2,3 core*2

2

2

\#O4 core

3

\#C5 core

4

\#N1=O2,3 bond pairs $* 4$

162745

162745

82945

82945 
\#O4-C5 bond

7345

\#C5-H6,7,8 bond

4579

4579

4597

\#O4-lone pair

7345162

94582

\#O2,3 lone pairs

216745

216745

216745

216745

\#Active VB Orbitals

12

37

\$end

\section{$\underline{\text { 5. } \mathrm{F}-\mathrm{NO}_{2}}$}

\section{Gamess-US input}

$\$$ contrl runtyp=energy $\mathrm{scftyp}=\mathrm{rhf}$ maxit $=200$ icharg $=0$ mult $=1$ vbtyp=xmvb $\$$ end $\$ \mathrm{scf}$ damp=.t. $\operatorname{soscf}=$.f. diis=.t. $\$$ end

\$system mwords $=125$ \$end

\$basis basnam $(1)=\mathrm{N}, \mathrm{O}, \mathrm{O}, \mathrm{F}$, \$end

\$data

$\mathrm{NO} 2 \mathrm{~F}$ at cc-pVDZ basis set

$\mathrm{C} 1$

$\begin{array}{lrrrr}\text { NITROGEN } & 7.0 & 0.000000000 & 0.000000000 & 0.000000000 \\ \text { OXYGEN } & 8.0 & -1.095092000 & 0.000000000 & -0.438782000 \\ \text { OXYGEN } & 8.0 & 1.095092000 & 0.000000000 & -0.438782000 \\ \text { FLUORINE } & 9.0 & 0.000000000 & 0.000000000 & 1.481802000\end{array}$

\$end

\$N

S 9

$1 \quad 9.046000 \mathrm{E}+03 \quad 7.000000 \mathrm{E}-04$

$2 \quad 1.357000 \mathrm{E}+03 \quad 5.389000 \mathrm{E}-03$

$3 \quad 3.093000 \mathrm{E}+02 \quad 2.740600 \mathrm{E}-02$

$4 \quad 8.773000 \mathrm{E}+01 \quad 1.032070 \mathrm{E}-01$

$5 \quad 2.856000 \mathrm{E}+01 \quad 2.787230 \mathrm{E}-01$

$6 \quad 1.021000 \mathrm{E}+01 \quad 4.485400 \mathrm{E}-01$

$7 \quad 3.838000 \mathrm{E}+00 \quad 2.782380 \mathrm{E}-01$

$8 \quad 7.466000 \mathrm{E}-01 \quad 1.544000 \mathrm{E}-02$

$9 \quad 2.248000 \mathrm{E}-01 \quad-2.864000 \mathrm{E}-03$ 


$\begin{array}{llll}\mathrm{S} & 9 & & \\ 1 & & 9.046000 \mathrm{E}+03 & -1.530000 \mathrm{E}-04 \\ 2 & & 1.357000 \mathrm{E}+03 & -1.208000 \mathrm{E}-03 \\ 3 & & 3.093000 \mathrm{E}+02 & -5.992000 \mathrm{E}-03 \\ 4 & & 8.773000 \mathrm{E}+01 & -2.454400 \mathrm{E}-02 \\ 5 & & 2.856000 \mathrm{E}+01 & -6.745900 \mathrm{E}-02 \\ 6 & & 1.021000 \mathrm{E}+01 & -1.580780 \mathrm{E}-01 \\ 7 & & 3.838000 \mathrm{E}+00 & -1.218310 \mathrm{E}-01 \\ 8 & & 7.466000 \mathrm{E}-01 & 5.490030 \mathrm{E}-01 \\ 9 & & 2.248000 \mathrm{E}-01 & 5.788150 \mathrm{E}-01 \\ \mathrm{~S} & 1 & & \\ 1 & & 2.248000 \mathrm{E}-01 & 1.000000 \mathrm{E}+00 \\ \mathrm{P} & 4 & & \\ 1 & & 1.355000 \mathrm{E}+01 & 3.991900 \mathrm{E}-02 \\ 2 & & 2.917000 \mathrm{E}+00 & 2.171690 \mathrm{E}-01 \\ 3 & & 7.973000 \mathrm{E}-01 & 5.103190 \mathrm{E}-01 \\ 4 & & 2.185000 \mathrm{E}-01 & 4.622140 \mathrm{E}-01 \\ \mathrm{P} & 1 & & \\ 1 & & 2.185000 \mathrm{E}-01 & 1.000000 \mathrm{E}+00 \\ \mathrm{D} & 1 & & \\ 1 & & 8.170000 \mathrm{E}-01 & 1.0000000\end{array}$

\begin{tabular}{lccc}
\multicolumn{3}{l}{ \$END } \\
\$O & & \\
S & 9 & & \\
1 & & $1.172000 \mathrm{E}+04$ & $7.100000 \mathrm{E}-04$ \\
2 & & $1.759000 \mathrm{E}+03$ & $5.470000 \mathrm{E}-03$ \\
3 & & $4.008000 \mathrm{E}+02$ & $2.783700 \mathrm{E}-02$ \\
4 & & $1.137000 \mathrm{E}+02$ & $1.048000 \mathrm{E}-01$ \\
5 & & $3.703000 \mathrm{E}+01$ & $2.830620 \mathrm{E}-01$ \\
6 & & $1.327000 \mathrm{E}+01$ & $4.487190 \mathrm{E}-01$ \\
7 & & $5.025000 \mathrm{E}+00$ & $2.709520 \mathrm{E}-01$ \\
8 & & $1.013000 \mathrm{E}+00$ & $1.545800 \mathrm{E}-02$ \\
9 & & $3.023000 \mathrm{E}-01$ & $-2.585000 \mathrm{E}-03$ \\
$\mathrm{~S}$ & 9 & & \\
1 & & $1.172000 \mathrm{E}+04$ & $-1.600000 \mathrm{E}-04$ \\
2 & & $1.759000 \mathrm{E}+03$ & $-1.263000 \mathrm{E}-03$ \\
3 & & $4.008000 \mathrm{E}+02$ & $-6.267000 \mathrm{E}-03$ \\
4 & & $1.137000 \mathrm{E}+02$ & $-2.571600 \mathrm{E}-02$ \\
5 & & $3.703000 \mathrm{E}+01$ & $-7.092400 \mathrm{E}-02$ \\
6 & & $1.327000 \mathrm{E}+01$ & $-1.654110 \mathrm{E}-01$ \\
7 & & $5.025000 \mathrm{E}+00$ & $-1.169550 \mathrm{E}-01$ \\
8 & & $1.013000 \mathrm{E}+00$ & $5.573680 \mathrm{E}-01$ \\
9 & & $3.023000 \mathrm{E}-01$ & $5.727590 \mathrm{E}-01$ \\
S & 1 & & \\
1 & & $3.023000 \mathrm{E}-01$ & $1.000000 \mathrm{E}+00$ \\
$\mathrm{P}$ & 4 & & \\
1 & & $1.770000 \mathrm{E}+01$ & $4.301800 \mathrm{E}-02$ \\
2 & & $3.854000 \mathrm{E}+00$ & $2.289130 \mathrm{E}-01$ \\
3 & & $1.046000 \mathrm{E}+00$ & $5.087280 \mathrm{E}-01$
\end{tabular}




$\begin{array}{llll}4 & & 2.753000 \mathrm{E}-01 & 4.605310 \mathrm{E}-01 \\ \mathrm{P} & 1 & & \\ 1 & & 2.753000 \mathrm{E}-01 & 1.000000 \mathrm{E}+00 \\ \mathrm{D} & 1 & & \\ 1 & & 1.185000 \mathrm{E}+00 & 1.0000000\end{array}$

\begin{tabular}{|c|c|c|}
\hline \multicolumn{3}{|c|}{ \$END } \\
\hline \multicolumn{3}{|c|}{$\$ F$} \\
\hline \multicolumn{3}{|c|}{ S 9} \\
\hline 1 & $1.471000 \mathrm{E}+04$ & 7.210000E-04 \\
\hline 2 & $2.207000 \mathrm{E}+03$ & $5.553000 \mathrm{E}-03$ \\
\hline 3 & $5.028000 \mathrm{E}+02$ & $2.826700 \mathrm{E}-02$ \\
\hline 4 & $1.426000 \mathrm{E}+02$ & $1.064440 \mathrm{E}-01$ \\
\hline 5 & $4.647000 \mathrm{E}+01$ & $2.868140 \mathrm{E}-01$ \\
\hline 6 & $1.670000 \mathrm{E}+01$ & 4.486410E-01 \\
\hline 7 & $6.356000 \mathrm{E}+00$ & $2.647610 \mathrm{E}-01$ \\
\hline 8 & $1.316000 \mathrm{E}+00$ & $1.533300 \mathrm{E}-02$ \\
\hline 9 & $3.897000 \mathrm{E}-01$ & $-2.332000 \mathrm{E}-03$ \\
\hline \multicolumn{3}{|c|}{ S 9} \\
\hline 1 & $1.471000 \mathrm{E}+04$ & $-1.650000 \mathrm{E}-04$ \\
\hline 2 & $2.207000 \mathrm{E}+03$ & $-1.308000 \mathrm{E}-03$ \\
\hline 3 & $5.028000 \mathrm{E}+02$ & $-6.495000 \mathrm{E}-03$ \\
\hline 4 & $1.426000 \mathrm{E}+02$ & $-2.669100 \mathrm{E}-02$ \\
\hline 5 & $4.647000 \mathrm{E}+01$ & $-7.369000 \mathrm{E}-02$ \\
\hline 6 & $1.670000 \mathrm{E}+01$ & $-1.707760 \mathrm{E}-01$ \\
\hline 7 & $6.356000 \mathrm{E}+00$ & $-1.123270 \mathrm{E}-01$ \\
\hline 8 & $1.316000 \mathrm{E}+00$ & $5.628140 \mathrm{E}-01$ \\
\hline 9 & $3.897000 \mathrm{E}-01$ & $5.687780 \mathrm{E}-01$ \\
\hline \multicolumn{3}{|c|}{ S 1} \\
\hline 1 & $3.897000 \mathrm{E}-01$ & $1.000000 \mathrm{E}+00$ \\
\hline \multicolumn{3}{|c|}{$\mathrm{P} 4$} \\
\hline 1 & $2.267000 \mathrm{E}+01$ & 4.487800E-02 \\
\hline 2 & $4.977000 \mathrm{E}+00$ & $2.357180 \mathrm{E}-01$ \\
\hline 3 & $1.347000 \mathrm{E}+00$ & $5.085210 \mathrm{E}-01$ \\
\hline 4 & $3.471000 \mathrm{E}-01$ & $4.581200 \mathrm{E}-01$ \\
\hline $\mathrm{P}$ & & \\
\hline 1 & $3.471000 \mathrm{E}-01$ & $1.000000 \mathrm{E}+00$ \\
\hline $\mathrm{D}$ & & \\
\hline 1 & $1.640000 \mathrm{E}+00$ & 1.0000000 \\
\hline
\end{tabular}

\section{$\underline{\text { XMVB input }}$}

NO2F cc-pVDZ

\$ctrl 
itmax $=2000$ nstr $=3$ nao $=2$ nae $=2$ iscf $=2$ guess $=$ read bovb nmul $=1$ orbtyp $=$ hao frgtyp $=$ sao iprint $=3$

Send

\$struct

$1: 151617$

$1: 151616$

$1: 151717$

Send

\$rag

1211111

SPzDxxDyyDzz 1

SPxPyPzDxxDyyDzzDxyDxzDyz 23

SPzDxxDyyDzz 4

PxDxz 1

PxDxz 4

PyDyzDxy 1

PyDyzDxy 4

\$end

\$orb

$1 * 44 * 23 * 24 * 414321$

\#N1 core

1

\#O2,3 Core

2

2

\#F4 core

3

$\# \mathrm{~N}=\mathrm{O}$ bond pairs $* 4$

1425

1425

627

627

\#O2,3 lone pairs

2145

2145

2145

2145

\#F4 lone pairs*3

3

5421

762

\#Active VB Orbitals

12

3

\$end 


\section{$\underline{\text { 6. } \mathrm{H}_{3} \mathrm{C}-\mathrm{CH}_{3}}$}

\section{Gamess-US input}

$\$$ contrl runtyp=energy scftyp $=$ rhf maxit $=200$ icharg $=0$ mult $=1$ vbtyp=xmvb \$end $\$ \mathrm{scf}$ damp=.t. $\operatorname{soscf}=$.f. diis=.t. $\$$ end

\$system mwords $=125$ Send

\$basis basnam $(1)=\mathrm{C}, \mathrm{C}, \mathrm{H}, \mathrm{H}, \mathrm{H}, \mathrm{H}, \mathrm{H}, \mathrm{H}$, Send

\$data

$\mathrm{C} 2 \mathrm{H} 4$ at $6-31 \mathrm{~g}(\mathrm{~d}, \mathrm{p})$ basis set

$\mathrm{C} 1$

$\begin{array}{lrrrr}\text { CARBON } & 6.0 & 0.000000000 & 0.000000000 & 0.762610000 \\ \text { CARBON } & 6.0 & 0.000000000 & 0.000000000 & -0.762610000 \\ \text { HYDROGEN } & 1.0 & 0.000000000 & 1.014907000 & 1.157941000 \\ \text { HYDROGEN } & 1.0 & -0.878936000 & -0.507454000 & 1.157941000 \\ \text { HYDROGEN } & 1.0 & 0.878936000 & -0.507454000 & 1.157941000 \\ \text { HYDROGEN } & 1.0 & 0.878936000 & 0.507454000 & -1.157941000 \\ \text { HYDROGEN } & 1.0 & 0.000000000 & -1.014907000 & -1.157941000 \\ \text { HYDROGEN } & 1.0 & -0.878936000 & 0.507454000 & -1.157941000\end{array}$

Send

\begin{tabular}{llll}
\multicolumn{2}{c}{$\$ \mathrm{H}$} & & \\
$\mathrm{S}$ & 4 & & \\
1 & & $1.301000 \mathrm{E}+01$ & $1.968500 \mathrm{E}-02$ \\
2 & & $1.962000 \mathrm{E}+00$ & $1.379770 \mathrm{E}-01$ \\
3 & & $4.446000 \mathrm{E}-01$ & $4.781480 \mathrm{E}-01$ \\
4 & & $1.220000 \mathrm{E}-01$ & $5.012400 \mathrm{E}-01$ \\
$\mathrm{~S}$ & 1 & & \\
1 & & $1.220000 \mathrm{E}-01$ & $1.000000 \mathrm{E}+00$ \\
$\mathrm{P}$ & 1 & & \\
1 & & $7.270000 \mathrm{E}-01$ & 1.0000000
\end{tabular}

\begin{tabular}{|c|c|c|}
\hline \multicolumn{3}{|c|}{ \$END } \\
\hline \multicolumn{3}{|c|}{$\$ C$} \\
\hline \multicolumn{3}{|c|}{ S 9} \\
\hline 1 & $6.665000 \mathrm{E}+03$ & $6.920000 \mathrm{E}-04$ \\
\hline 2 & $1.000000 \mathrm{E}+03$ & $5.329000 \mathrm{E}-03$ \\
\hline 3 & $2.280000 \mathrm{E}+02$ & $2.707700 \mathrm{E}-02$ \\
\hline 4 & $6.471000 \mathrm{E}+01$ & $1.017180 \mathrm{E}-01$ \\
\hline 5 & $2.106000 \mathrm{E}+01$ & $2.747400 \mathrm{E}-01$ \\
\hline 6 & $7.495000 \mathrm{E}+00$ & $4.485640 \mathrm{E}-01$ \\
\hline 7 & $2.797000 \mathrm{E}+00$ & $2.850740 \mathrm{E}-01$ \\
\hline 8 & $5.215000 \mathrm{E}-01$ & $1.520400 \mathrm{E}-02$ \\
\hline 9 & $1.596000 \mathrm{E}-01$ & $-3.191000 \mathrm{E}-03$ \\
\hline \\
\hline 1 & $6.665000 \mathrm{E}+03$ & $-1.460000 \mathrm{E}-04$ \\
\hline 2 & $1.000000 \mathrm{E}+03$ & $-1.154000 \mathrm{E}-03$ \\
\hline 3 & $2.280000 \mathrm{E}+02$ & $-5.725000 \mathrm{E}-03$ \\
\hline & $6.471000 \mathrm{E}+01$ & $-2.331200 \mathrm{E}-02$ \\
\hline & $2.106000 \mathrm{E}+01$ & $-6.395500 \mathrm{E}-02$ \\
\hline
\end{tabular}




$\begin{array}{llll}6 & & 7.495000 \mathrm{E}+00 & -1.499810 \mathrm{E}-01 \\ 7 & & 2.797000 \mathrm{E}+00 & -1.272620 \mathrm{E}-01 \\ 8 & & 5.215000 \mathrm{E}-01 & 5.445290 \mathrm{E}-01 \\ 9 & & 1.596000 \mathrm{E}-01 & 5.804960 \mathrm{E}-01 \\ \mathrm{~S} & 1 & & \\ 1 & & 1.596000 \mathrm{E}-01 & 1.000000 \mathrm{E}+00 \\ \mathrm{P} & 4 & & \\ 1 & & 9.439000 \mathrm{E}+00 & 3.810900 \mathrm{E}-02 \\ 2 & & 2.002000 \mathrm{E}+00 & 2.094800 \mathrm{E}-01 \\ 3 & & 5.456000 \mathrm{E}-01 & 5.085570 \mathrm{E}-01 \\ 4 & & 1.517000 \mathrm{E}-01 & 4.688420 \mathrm{E}-01 \\ \mathrm{P} & 1 & & \\ 1 & & 1.517000 \mathrm{E}-01 & 1.000000 \mathrm{E}+00 \\ \mathrm{D} & 1 & & \\ 1 & & 5.500000 \mathrm{E}-01 & 1.0000000\end{array}$

\begin{tabular}{|c|c|c|c|}
\hline \multicolumn{4}{|c|}{ \$END } \\
\hline \multicolumn{4}{|c|}{ \$N } \\
\hline \multicolumn{4}{|c|}{ S 9} \\
\hline 1 & & $9.046000 \mathrm{E}+03$ & $7.000000 \mathrm{E}-04$ \\
\hline 2 & & $1.357000 \mathrm{E}+03$ & $5.389000 \mathrm{E}-03$ \\
\hline 3 & & $3.093000 \mathrm{E}+02$ & $2.740600 \mathrm{E}-02$ \\
\hline 4 & & $8.773000 \mathrm{E}+01$ & $1.032070 \mathrm{E}-01$ \\
\hline 5 & & $2.856000 \mathrm{E}+01$ & 2.787230E-01 \\
\hline 6 & & $1.021000 \mathrm{E}+01$ & $4.485400 \mathrm{E}-01$ \\
\hline 7 & & $3.838000 \mathrm{E}+00$ & $2.782380 \mathrm{E}-01$ \\
\hline 8 & & $7.466000 \mathrm{E}-01$ & $1.544000 \mathrm{E}-02$ \\
\hline 9 & & $2.248000 \mathrm{E}-01$ & $-2.864000 \mathrm{E}-03$ \\
\hline \multicolumn{4}{|c|}{ S 9} \\
\hline 1 & & $9.046000 \mathrm{E}+03$ & $-1.530000 \mathrm{E}-04$ \\
\hline 2 & & $1.357000 \mathrm{E}+03$ & $-1.208000 \mathrm{E}-03$ \\
\hline 3 & & $3.093000 \mathrm{E}+02$ & $-5.992000 \mathrm{E}-03$ \\
\hline 4 & & $8.773000 \mathrm{E}+01$ & $-2.454400 \mathrm{E}-02$ \\
\hline 5 & & $2.856000 \mathrm{E}+01$ & $-6.745900 \mathrm{E}-02$ \\
\hline 6 & & $1.021000 \mathrm{E}+01$ & $-1.580780 \mathrm{E}-01$ \\
\hline 7 & & $3.838000 \mathrm{E}+00$ & $-1.218310 \mathrm{E}-01$ \\
\hline 8 & & $7.466000 \mathrm{E}-01$ & $5.490030 \mathrm{E}-01$ \\
\hline 9 & & $2.248000 \mathrm{E}-01$ & $5.788150 \mathrm{E}-01$ \\
\hline \multicolumn{4}{|c|}{$\begin{array}{ll}S & 1\end{array}$} \\
\hline 1 & & $2.248000 \mathrm{E}-01$ & $1.000000 \mathrm{E}+00$ \\
\hline \multicolumn{4}{|c|}{ P 4} \\
\hline 1 & & $1.355000 \mathrm{E}+01$ & 3.991900E-02 \\
\hline 2 & & $2.917000 \mathrm{E}+00$ & $2.171690 \mathrm{E}-01$ \\
\hline 3 & & $7.973000 \mathrm{E}-01$ & $5.103190 \mathrm{E}-01$ \\
\hline 4 & & $2.185000 \mathrm{E}-01$ & 4.622140E-01 \\
\hline \multicolumn{4}{|c|}{$\mathrm{P} \quad 1$} \\
\hline 1 & & $2.185000 \mathrm{E}-01$ & $1.000000 \mathrm{E}+00$ \\
\hline $\mathrm{D}$ & 1 & & \\
\hline 1 & & $8.170000 \mathrm{E}-01$ & 1.0000000 \\
\hline
\end{tabular}




\section{$\underline{\text { XMVB input }}$}

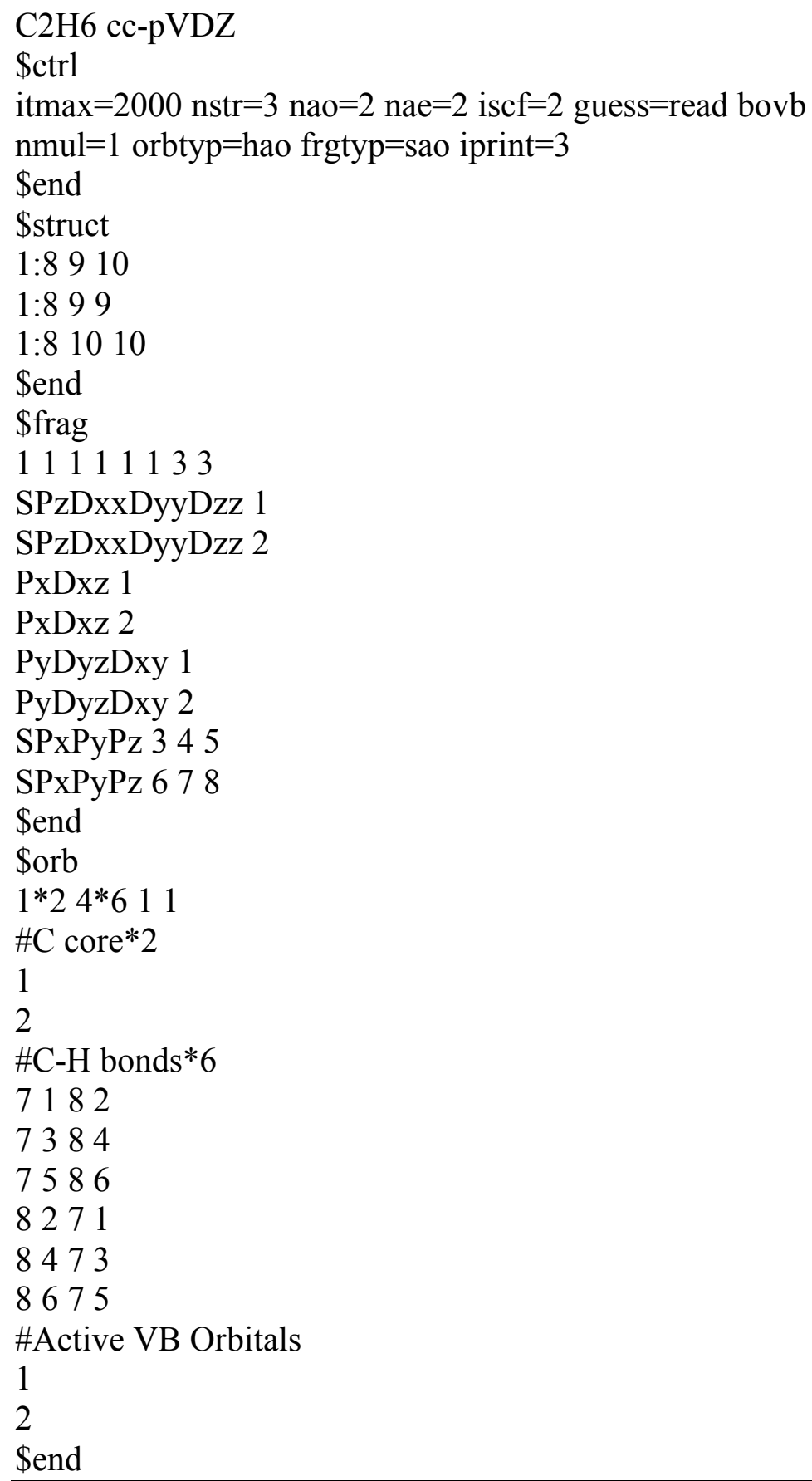

\section{7. $\mathrm{H}_{3} \mathrm{C}-\mathrm{NH}_{2}$}

\section{Gamess-US input}

$\$$ contrl runtyp $=$ energy $\mathrm{scfty} p=\mathrm{rhf}$ maxit $=200$ icharg $=0$ mult $=1$ vbtyp $=\mathrm{xmvb}$ \$end $\$ \mathrm{scf}$ damp=.t. $\operatorname{soscf}=$.f. diis=.t. $\$$ end \$system mwords $=125$ \$end \$basis basnam (1) $=\mathrm{C}, \mathrm{H}, \mathrm{H}, \mathrm{H}, \mathrm{N}, \mathrm{H}, \mathrm{H}$, Send 
\$data

MethylAmine at cc-pVDZ basis set

C1

$\begin{array}{lrrrr}\text { CARBON } & 6.0 & 0.000000000 & 0.000000000 & 0.730233500 \\ \text { HYDROGEN } & 1.0 & -0.538819000 & 0.876314000 & 1.088163500 \\ \text { HYDROGEN } & 1.0 & -0.538819000 & -0.876314000 & 1.088163500 \\ \text { HYDROGEN } & 1.0 & 0.992158000 & 0.000000000 & 1.194516500 \\ \text { NITROGEN } & 7.0 & 0.000000000 & 0.000000000 & -0.730233500 \\ \text { HYDROGEN } & 1.0 & 0.495325000 & 0.809964000 & -1.079911500 \\ \text { HYDROGEN } & 1.0 & 0.495325000 & -0.809964000 & -1.079911500\end{array}$

Send

\begin{tabular}{llll}
\multicolumn{2}{c}{$\$ \mathrm{H}$} & & \\
$\mathrm{S}$ & 4 & & \\
1 & & $1.301000 \mathrm{E}+01$ & $1.968500 \mathrm{E}-02$ \\
2 & & $1.962000 \mathrm{E}+00$ & $1.379770 \mathrm{E}-01$ \\
3 & & $4.446000 \mathrm{E}-01$ & $4.781480 \mathrm{E}-01$ \\
4 & & $1.220000 \mathrm{E}-01$ & $5.012400 \mathrm{E}-01$ \\
$\mathrm{~S}$ & 1 & & \\
1 & & $1.220000 \mathrm{E}-01$ & $1.000000 \mathrm{E}+00$ \\
$\mathrm{P}$ & 1 & & \\
1 & & $7.270000 \mathrm{E}-01$ & 1.0000000
\end{tabular}

\begin{tabular}{|c|c|c|c|}
\hline \multicolumn{4}{|c|}{ \$END } \\
\hline \multicolumn{4}{|c|}{$\$ \mathrm{C}$} \\
\hline \multicolumn{4}{|c|}{ S 9} \\
\hline 1 & & $6.665000 \mathrm{E}+03$ & $6.920000 \mathrm{E}-04$ \\
\hline 2 & & $1.000000 \mathrm{E}+03$ & $5.329000 \mathrm{E}-03$ \\
\hline 3 & & $2.280000 \mathrm{E}+02$ & $2.707700 \mathrm{E}-02$ \\
\hline 4 & & $6.471000 \mathrm{E}+01$ & $1.017180 \mathrm{E}-01$ \\
\hline 5 & & $2.106000 \mathrm{E}+01$ & $2.747400 \mathrm{E}-01$ \\
\hline 6 & & $7.495000 \mathrm{E}+00$ & 4.485640E-01 \\
\hline 7 & & $2.797000 \mathrm{E}+00$ & $2.850740 \mathrm{E}-01$ \\
\hline 8 & & $5.215000 \mathrm{E}-01$ & $1.520400 \mathrm{E}-02$ \\
\hline 9 & & $1.596000 \mathrm{E}-01$ & $-3.191000 \mathrm{E}-03$ \\
\hline S & \multicolumn{3}{|r|}{ S.1800 } \\
\hline 1 & & $6.665000 \mathrm{E}+03$ & $-1.460000 \mathrm{E}-04$ \\
\hline 2 & & $1.000000 \mathrm{E}+03$ & $-1.154000 \mathrm{E}-03$ \\
\hline 3 & & $2.280000 \mathrm{E}+02$ & $-5.725000 \mathrm{E}-03$ \\
\hline 4 & & $6.471000 \mathrm{E}+01$ & $-2.331200 \mathrm{E}-02$ \\
\hline 5 & & $2.106000 \mathrm{E}+01$ & $-6.395500 \mathrm{E}-02$ \\
\hline 6 & & $7.495000 \mathrm{E}+00$ & $-1.499810 \mathrm{E}-01$ \\
\hline 7 & & $2.797000 \mathrm{E}+00$ & $-1.272620 \mathrm{E}-01$ \\
\hline 8 & & $5.215000 \mathrm{E}-01$ & $5.445290 \mathrm{E}-01$ \\
\hline 9 & & $1.596000 \mathrm{E}-01$ & $5.804960 \mathrm{E}-01$ \\
\hline S & 1 & & \\
\hline 1 & & $1.596000 \mathrm{E}-01$ & $1.000000 \mathrm{E}+00$ \\
\hline $\mathrm{P}$ & 4 & & \\
\hline 1 & & $9.439000 \mathrm{E}+00$ & 3.810900E-02 \\
\hline 2 & & $2.002000 \mathrm{E}+00$ & $2.094800 \mathrm{E}-01$ \\
\hline
\end{tabular}




$\begin{array}{llll}3 & & 5.456000 \mathrm{E}-01 & 5.085570 \mathrm{E}-01 \\ 4 & & 1.517000 \mathrm{E}-01 & 4.688420 \mathrm{E}-01 \\ \mathrm{P} & 1 & & \\ 1 & & 1.517000 \mathrm{E}-01 & 1.000000 \mathrm{E}+00 \\ \mathrm{D} & 1 & & \\ 1 & & 5.500000 \mathrm{E}-01 & 1.0000000\end{array}$

\begin{tabular}{llll}
\multicolumn{3}{l}{ \$END } & \\
\$N & & \\
S & 9 & & \\
1 & & $9.046000 \mathrm{E}+03$ & $7.000000 \mathrm{E}-04$ \\
2 & & $1.357000 \mathrm{E}+03$ & $5.389000 \mathrm{E}-03$ \\
3 & & $3.093000 \mathrm{E}+02$ & $2.740600 \mathrm{E}-02$ \\
4 & & $8.773000 \mathrm{E}+01$ & $1.032070 \mathrm{E}-01$ \\
5 & & $2.856000 \mathrm{E}+01$ & $2.787230 \mathrm{E}-01$ \\
6 & & $1.021000 \mathrm{E}+01$ & $4.485400 \mathrm{E}-01$ \\
7 & & $3.838000 \mathrm{E}+00$ & $2.782380 \mathrm{E}-01$ \\
8 & & $7.466000 \mathrm{E}-01$ & $1.544000 \mathrm{E}-02$ \\
9 & & $2.248000 \mathrm{E}-01$ & $-2.864000 \mathrm{E}-03$ \\
$\mathrm{~S}$ & 9 & & \\
1 & & $9.046000 \mathrm{E}+03$ & $-1.530000 \mathrm{E}-04$ \\
2 & & $1.357000 \mathrm{E}+03$ & $-1.208000 \mathrm{E}-03$ \\
3 & & $3.093000 \mathrm{E}+02$ & $-5.992000 \mathrm{E}-03$ \\
4 & & $8.773000 \mathrm{E}+01$ & $-2.454400 \mathrm{E}-02$ \\
5 & & $2.856000 \mathrm{E}+01$ & $-6.745900 \mathrm{E}-02$ \\
6 & & $1.021000 \mathrm{E}+01$ & $-1.580780 \mathrm{E}-01$ \\
7 & & $3.838000 \mathrm{E}+00$ & $-1.218310 \mathrm{E}-01$ \\
8 & & $7.466000 \mathrm{E}-01$ & $5.490030 \mathrm{E}-01$ \\
9 & & $2.248000 \mathrm{E}-01$ & $5.788150 \mathrm{E}-01$ \\
$\mathrm{~S}$ & 1 & & \\
1 & & $2.248000 \mathrm{E}-01$ & $1.000000 \mathrm{E}+00$ \\
$\mathrm{P}$ & 4 & & \\
1 & & $1.355000 \mathrm{E}+01$ & $3.991900 \mathrm{E}-02$ \\
2 & & $2.917000 \mathrm{E}+00$ & $2.171690 \mathrm{E}-01$ \\
3 & & $7.973000 \mathrm{E}-01$ & $5.103190 \mathrm{E}-01$ \\
4 & & $2.185000 \mathrm{E}-01$ & $4.622140 \mathrm{E}-01$ \\
$\mathrm{P}$ & 1 & & \\
1 & & $2.185000 \mathrm{E}-01$ & $1.000000 \mathrm{E}+00$ \\
$\mathrm{D}$ & 1 & & \\
1 & & $8.170000 \mathrm{E}-01$ & 1.0000000 \\
& &
\end{tabular}

\section{\$END}

\section{$\underline{\text { XMVB input }}$}

MethylAmine cc-pVDZ

\$ctrl

itmax $=2000$ nstr $=3$ nao $=2$ nae $=2$ isc $=2$ guess $=$ read bovb nmul $=1$ orbtyp $=$ hao frgtyp $=$ sao iprint $=3$

\$end 


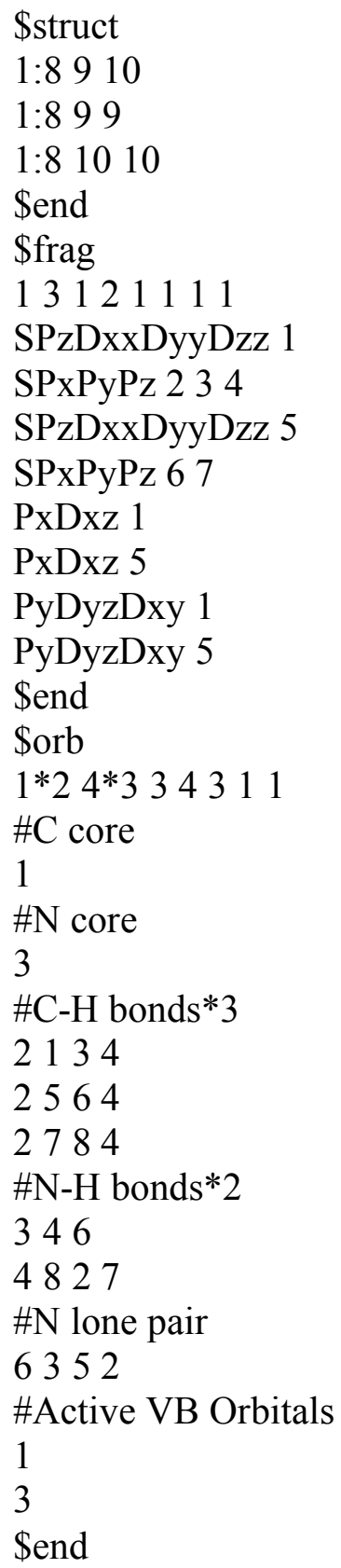

\section{Cartesian Coordinates of the Compounds}

All structures are optimized at the B2PLYP-D3/cc-pVTZ level of theory using Gaussian-16.

\section{1. $\mathrm{H}-\mathrm{NO}_{2}$}

Number of Imaginary Frequencies $=0$

Zero-point correction $=$

Thermal correction to Energy $=$

Thermal correction to Enthalpy $=$ 0.021825 (Hartree/Particle)

0.024787

0.025731 


\begin{tabular}{|c|c|c|c|c|}
\hline & 1 correction & Gibbs Free En & & $-0.0 c$ \\
\hline & f electronic ar & zero-point Ene & gies $=$ & 2 \\
\hline & f electronic ar & thermal Energi & & \\
\hline & f electronic ar & thermal Enthal] & ies $=$ & \\
\hline & of electronic ar & thermal Free E & ergies $=$ & \\
\hline $\mathrm{N}$ & 0.000000000 & 0.000000000 & 0.31 & 374000 \\
\hline $\mathrm{O}$ & 0.000000000 & 1.097977000 & -0.21 & 971000 \\
\hline $\mathrm{O}$ & 0.000000000 & -1.097977000 & -0.21 & 9971000 \\
\hline $\mathrm{H}$ & 0.000000000 & 0.000000000 & 1.3 & \\
\hline
\end{tabular}

\section{2. $\mathrm{H}_{3} \mathrm{C}-\mathrm{NO}_{2}$}

Number of Imaginary Frequencies $=0$

Zero-point correction $=$ 0.049875 (Hartree/Particle)

Thermal correction to Energy $=$

Thermal correction to Enthalpy =

Thermal correction to Gibbs Free Energy = Sum of electronic and zero-point Energies $=$

Sum of electronic and thermal Energies $=$

Sum of electronic and thermal Enthalpies =

Sum of electronic and thermal Free Energies $=\quad-244.916131$
$\mathrm{N} \quad-0.172046000 \quad 0.000001000 \quad-0.009285000$
$\begin{array}{llll}\text { O } & -0.729074000 & -1.089897000 & 0.002524000\end{array}$
$\begin{array}{llll}\text { O } & -0.728819000 & 1.090030000 & 0.002524000\end{array}$
C $\quad 1.320653000 \quad-0.000125000 \quad-0.003059000$
$\mathrm{H} \quad 1.629410000 \quad 0.000938000 \quad 1.037843000$
$\mathrm{H} \quad 1.657015000 \quad-0.902617000 \quad-0.496550000$
H $\quad 1.657128000 \quad 0.901360000 \quad-0.498324000$

\section{3. $\left(\mathrm{H}_{3} \mathrm{C}\right)_{2} \mathrm{~N}-\mathrm{NO}_{2}$}

Number of Imaginary Frequencies $=0$

Zero-point correction $=$ 0.095464 (Hartree/Particle)

Thermal correction to Energy =

$$
\begin{gathered}
0.053348 \\
0.054292 \\
0.024077 \\
-244.890333 \\
-244.886860 \\
-244.885916
\end{gathered}
$$$$
-205.647478
$$

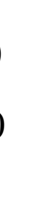




\begin{tabular}{|c|c|c|c|c|}
\hline & electronic & ero-point E & ies $=$ & -3 \\
\hline & of electronic ar & thermal Energi & es $=$ & -33 \\
\hline & of electronic a & thermal Enthal & bies $=$ & \\
\hline & of electronic ar & 1 thermal Free $\mathrm{E}$ & nergies $=$ & \\
\hline & -0.565236000 & 0.000000000 & -0.25221 & \\
\hline & 0.800511000 & 0.000000000 & -0.03322 & \\
\hline & 1.353538000 & -1.093358000 & 0.03636 & \\
\hline & 1.353538000 & 1.093358000 & 0.036361 & \\
\hline & -1.235214000 & 1.253872000 & 0.0560 & \\
\hline & -0.774160000 & 2.062130000 & -0.4974 & \\
\hline & -2.270249000 & 1.147936000 & -0.2532 & \\
\hline & -1.196078000 & 1.487690000 & 1.1224 & \\
\hline & -1.235214000 & -1.253872000 & 0.05606 & \\
\hline & -2.270249000 & -1.147936000 & -0.2532 & \\
\hline & -0.774161000 & -2.062130000 & -0.4974 & \\
\hline & -1.196078000 & -1.487690000 & 1.12246 & 00 \\
\hline
\end{tabular}

\section{4. $\left(\mathrm{H}_{3} \mathrm{C}\right) \mathrm{O}-\mathrm{NO}_{2}$}

Number of Imaginary Frequencies $=0$

Zero-point correction $=$ 0.054470 (Hartree/Particle)

Thermal correction to Energy $=$ 0.059415

Thermal correction to Enthalpy $=$ 0.060359

Thermal correction to Gibbs Free Energy $=0.026468$

Sum of electronic and zero-point Energies $=\quad-320.050925$

Sum of electronic and thermal Energies $=\quad-320.045981$

Sum of electronic and thermal Enthalpies $=\quad-320.045037$

Sum of electronic and thermal Free Energies $=\quad-320.078928$
$\mathrm{N} \quad-0.619632000 \quad 0.051054000 \quad 0.000000000$
$\begin{array}{llll}\mathrm{O} & -0.454411000 & 1.249222000 & 0.000000000\end{array}$
$\begin{array}{llll}\mathrm{O} & -1.634587000 & -0.590608000 & 0.000001000\end{array}$
$\begin{array}{llll}\text { O } & 0.541330000 & -0.763480000 & -0.000002000\end{array}$
$\begin{array}{llll}\text { C } & 1.755735000 & 0.000471000 & 0.000001000\end{array}$ 


$\begin{array}{llll}\mathrm{H} & 1.827135000 & 0.619032000 & 0.889915000 \\ \mathrm{H} & 2.530077000 & -0.759340000 & 0.000004000 \\ \mathrm{H} & 1.827140000 & 0.619031000 & -0.889914000\end{array}$

\section{5. $\mathrm{F}-\mathrm{NO}_{2}$}

Number of Imaginary Frequencies $=0$

Zero-point correction $=$ 0.013477 (Hartree/Particle)

Thermal correction to Energyz = 0.016844

Thermal correction to Enthalpy = 0.017788

Thermal correction to Gibbs Free Energy $=\quad-0.012294$

Sum of electronic and zero-point Energies $=$ $-304.818535$

Sum of electronic and thermal Energies $=$ $-304.815167$

Sum of electronic and thermal Enthalpies = $-304.814223$

Sum of electronic and thermal Free Energies $=\quad-304.844305$
$\begin{array}{llll}\mathrm{N} & 0.000000000 & -0.197366000 & 0.000000000\end{array}$
$\begin{array}{llll}\text { O } & 0.000000000 & -0.636148000 & 1.095092000\end{array}$
$\begin{array}{llll}\text { O } & 0.000000000 & -0.636148000 & -1.095092000\end{array}$
F $\quad 0.000000000 \quad 1.284436000 \quad 0.000000000$

\section{6. $\mathrm{H}_{3} \mathrm{C}-\mathrm{CH}_{3}$}

Number of Imaginary Frequencies $=0$

\begin{tabular}{|c|c|c|c|c|}
\hline \multicolumn{3}{|c|}{ Zero-point correction $=$} & \multicolumn{2}{|c|}{0.075094 (Hart } \\
\hline \multicolumn{3}{|c|}{ Thermal correction to Energy $=$} & \multicolumn{2}{|c|}{0.078570} \\
\hline \multicolumn{3}{|c|}{ Thermal correction to Enthalpy $=$} & & .079515 \\
\hline \multicolumn{5}{|c|}{ Thermal correction to Gibbs Free Energy = } \\
\hline \multicolumn{5}{|c|}{ Sum of electronic and zero-point Energies $=$} \\
\hline \multicolumn{5}{|c|}{ Sum of electronic and thermal Energies $=$} \\
\hline \multicolumn{5}{|c|}{ Sum of electronic and thermal Enthalpies $=$} \\
\hline \multicolumn{5}{|c|}{ Sum of electronic and thermal Free Energies $=$} \\
\hline & 0.000000000 & 0.000000000 & 0.7 & 10000 \\
\hline & 0.000000000 & 1.014907000 & 1.1 & 41000 \\
\hline & -0.878936000 & -0.507454000 & & 941000 \\
\hline & 0.878936000 & -0.507454000 & & 941000 \\
\hline & 0.878936000 & 0.507454000 & -1.1 & 941000 \\
\hline & 0.000000000 & -1.014907000 & -1.15 & 94100 \\
\hline
\end{tabular}



$\mathrm{H} \quad-0.878936000 \quad 0.507454000 \quad-1.157941000$
C $\quad 0.000000000 \quad 0.000000000 \quad-0.762610000$

\section{7. $\mathrm{H}_{3} \mathrm{C}-\mathrm{NH}_{2}$}

Number of Imaginary Frequencies $=0$

\begin{tabular}{|c|c|c|c|c|}
\hline \multicolumn{3}{|c|}{ Zero-point correction $=$} & \multicolumn{2}{|c|}{0.064410 (Hartree/Particle } \\
\hline \multicolumn{3}{|c|}{ Thermal correction to Energy $=$} & \multicolumn{2}{|c|}{0.067831} \\
\hline \multicolumn{3}{|c|}{ Thermal correction to Enthalpy $=$} & \multicolumn{2}{|c|}{0.068775} \\
\hline \multicolumn{4}{|c|}{ Thermal correction to Gibbs Free Energy $=$} & 0.041491 \\
\hline \multicolumn{4}{|c|}{ Sum of electronic and zero-point Energies $=$} & -95.743942 \\
\hline \multicolumn{4}{|c|}{ Sum of electronic and thermal Energies $=$} & -95.740521 \\
\hline \multicolumn{4}{|c|}{ Sum of electronic and thermal Enthalpies $=$} & -95.739576 \\
\hline \multicolumn{4}{|c|}{ Sum of electronic and thermal Free Energies $=$} & -95.766860 \\
\hline $\mathrm{C}$ & 0.050726000 & 0.704491000 & 0.0000 & 000 \\
\hline $\mathrm{H}$ & 0.589131000 & 1.062092000 & 0.8758 & 5000 \\
\hline 1 & 0.589131000 & 1.062092000 & -0.8758 & 5000 \\
\hline & -0.940067000 & 1.171492000 & 0.0000 & 0000 \\
\hline N & 0.050726000 & -0.758944000 & 0.0000 & 0000 \\
\hline & -0.448813000 & -1.105007000 & 0.8083 & 4000 \\
\hline $\mathrm{H}$ & -0.448813000 & -1.105007000 & -0.808 & 4000 \\
\hline
\end{tabular}

All the following structures are optimized at the B3PLYP-D3(BJ)/cc-pVTZ level of theory using Gaussian-16.

\section{TATB}

Number of Imaginary Frequencies $=0$

Zero-point correction $=$

0.157944 (Hartree/Particle)

Thermal correction to Energy =

0.173047

Thermal correction to Enthalpy $=$

0.173991

Thermal correction to Gibbs Free Energy =

0.110228

Sum of electronic and zero-point Energies $=$

$-1012.118008$

Sum of electronic and thermal Energies $=$

$-1012.102905$ 


\begin{tabular}{|c|c|c|c|}
\hline \multicolumn{4}{|c|}{ Sum of electronic and thermal Enthalpies = } \\
\hline \multicolumn{4}{|c|}{ Sum of electronic and thermal Free Energies $=$} \\
\hline $\mathrm{C}$ & 0.051641000 & -1.444868000 & -0.038305000 \\
\hline & 1.264042000 & -0.671399000 & 0.027445000 \\
\hline$C$ & 1.226346000 & 0.767063000 & -0.009497000 \\
\hline & -0.051047000 & 1.429885000 & -0.011741000 \\
\hline & -1.276915000 & 0.677831000 & 0.044489000 \\
\hline & -1.213283000 & -0.759359000 & 0.001965000 \\
\hline & -2.427585000 & -1.518518000 & -0.010991000 \\
\hline & -2.402875000 & -2.749501000 & -0.166670000 \\
\hline 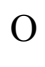 & -3.525720000 & -0.958746000 & 0.133594000 \\
\hline v & -2.442273000 & 1.297280000 & 0.118246000 \\
\hline 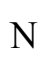 & -0.102481000 & 2.860543000 & -0.052345000 \\
\hline & 0.932572000 & 3.525829000 & -0.213662000 \\
\hline & -1.182850000 & 3.459398000 & 0.067568000 \\
\hline & 2.346822000 & 1.468528000 & -0.025895000 \\
\hline & 2.528517000 & -1.342400000 & 0.066366000 \\
\hline 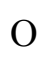 & 2.591837000 & -2.580297000 & 0.002681000 \\
\hline O & 3.583343000 & -0.697714000 & 0.175860000 \\
\hline J & 0.099038000 & -2.764207000 & -0.102926000 \\
\hline 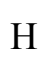 & -2.423544000 & 2.308286000 & 0.138974000 \\
\hline 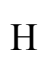 & -3.268882000 & 0.715962000 & 0.158064000 \\
\hline & 3.213292000 & 0.950226000 & 0.031686000 \\
\hline & 2.258191000 & 2.473274000 & -0.097781000 \\
\hline & 1.016391000 & -3.190211000 & -0.103138000 \\
\hline & -0.784883000 & -3.252775000 & -0.15608400 \\
\hline
\end{tabular}

\section{DATB}

Number of Imaginary Frequencies $=0$

Zero-point correction= 0.141648 (Hartree/Particle)

Thermal correction to Energy=

0.155555

Thermal correction to Enthalpy=

0.156499 


\begin{tabular}{|c|c|c|c|c|}
\hline \multicolumn{4}{|c|}{ Thermal correction to Gibbs Free Energy= } & 0.099073 \\
\hline \multicolumn{4}{|c|}{ Sum of electronic and zero-point Energies $=$} & -956.743529 \\
\hline \multicolumn{4}{|c|}{ Sum of electronic and thermal Energies= } & -956.729621 \\
\hline \multicolumn{4}{|c|}{ Sum of electronic and thermal Enthalpies= } & -956.728677 \\
\hline \multicolumn{4}{|c|}{ Sum of electronic and thermal Free Energies $=$} & -956.786103 \\
\hline & 1.260428000 & 0.588517000 & 0.0002 & 3000 \\
\hline & 1.203655000 & -0.844592000 & 0.0000 & 0000 \\
\hline & 0.000019000 & -1.506416000 & -0.0000 & 3000 \\
\hline & -1.203633000 & -0.844624000 & 0.0000 & 9000 \\
\hline & -1.260447000 & 0.588482000 & -0.0002 & 6000 \\
\hline & -0.000019000 & 1.287266000 & 0.0000 & 4000 \\
\hline & -0.000036000 & 2.724778000 & -0.0000 & 1000 \\
\hline & 1.070278000 & 3.343851000 & 0.0001 & 5000 \\
\hline & -1.070363000 & 3.343828000 & -0.0002 & 4000 \\
\hline & -2.429741000 & 1.210212000 & -0.0008 & 7000 \\
\hline & -2.390673000 & -1.683306000 & 0.0002 & 6000 \\
\hline & -2.241235000 & -2.893675000 & 0.0000 & 3000 \\
\hline & -3.507624000 & -1.148256000 & 0.0006 & 3000 \\
\hline & 2.390717000 & -1.683245000 & -0.0002 & 1000 \\
\hline & 3.507656000 & -1.148170000 & -0.0005 & 4000 \\
\hline & 2.241318000 & -2.893619000 & -0.0001 & 2000 \\
\hline & 2.429704000 & 1.210280000 & 0.0008 & 4000 \\
\hline & -3.255350000 & 0.625682000 & -0.0004 & 2000 \\
\hline & -2.430022000 & 2.218652000 & -0.0006 & 6000 \\
\hline & 3.255329000 & 0.625773000 & 0.0004 & 8000 \\
\hline & 2.429957000 & 2.218720000 & 0.0006 & 8000 \\
\hline & 0.000031000 & -2.583337000 & -0.0000 & 6000 \\
\hline
\end{tabular}

\section{MATB}

Number of Imaginary Frequencies $=0$

Zero-point correction=

0.124656 (Hartree/Particle)

Thermal correction to Energy=

0.137603

Thermal correction to Enthalpy=

0.138547 


\begin{tabular}{|c|c|c|c|c|}
\hline \multicolumn{4}{|c|}{ Thermal correction to Gibbs Free Energy= } & 0.083265 \\
\hline \multicolumn{4}{|c|}{ Sum of electronic and zero-point Energies= } & -901.362962 \\
\hline \multicolumn{4}{|c|}{ Sum of electronic and thermal Energies= } & -901.350015 \\
\hline \multicolumn{4}{|c|}{ Sum of electronic and thermal Enthalpies= } & -901.349071 \\
\hline \multicolumn{4}{|c|}{ Sum of electronic and thermal Free Energies= } & -901.404353 \\
\hline & -0.852476000 & -1.201545000 & \multicolumn{2}{|c|}{0.000016000} \\
\hline & -1.533624000 & -0.000013000 & \multicolumn{2}{|c|}{-0.000035000} \\
\hline & -0.852500000 & 1.201534000 & \multicolumn{2}{|c|}{-0.000007000} \\
\hline & 0.526182000 & 1.208094000 & \multicolumn{2}{|c|}{-0.000006000} \\
\hline & 1.301725000 & 0.000016000 & \multicolumn{2}{|c|}{-0.000062000} \\
\hline & 0.526209000 & -1.208079000 & \multicolumn{2}{|c|}{-0.000022000} \\
\hline & 1.158127000 & -2.530595000 & \multicolumn{2}{|c|}{0.000039000} \\
\hline & 0.436071000 & -3.509730000 & \multicolumn{2}{|c|}{0.000188000} \\
\hline & 2.390203000 & -2.596714000 & \multicolumn{2}{|c|}{-0.000045000} \\
\hline & 2.629089000 & 0.000032000 & \multicolumn{2}{|c|}{-0.000212000} \\
\hline & 1.158085000 & 2.530611000 & \multicolumn{2}{|c|}{0.000095000} \\
\hline & 0.436020000 & 3.509742000 & \multicolumn{2}{|c|}{0.000414000} \\
\hline & 2.390160000 & 2.596726000 & \multicolumn{2}{|c|}{-0.000158000} \\
\hline & -2.999019000 & -0.000027000 & \multicolumn{2}{|c|}{-0.000079000} \\
\hline & -3.559644000 & -1.085027000 & \multicolumn{2}{|c|}{0.000198000} \\
\hline & -3.559655000 & 1.084975000 & \multicolumn{2}{|c|}{-0.000346000} \\
\hline & 3.098956000 & 0.892333000 & \multicolumn{2}{|c|}{-0.000164000} \\
\hline & 3.098969000 & -0.892266000 & \multicolumn{2}{|c|}{-0.000123000} \\
\hline & -1.390131000 & 2.135094000 & \multicolumn{2}{|c|}{0.000000000} \\
\hline & -1.390093000 & -2.135112000 & \multicolumn{2}{|c|}{0.000072000} \\
\hline
\end{tabular}

\section{TNT}

Number of Imaginary Frequencies $=0$

Zero-point correction $=$ 0.135134 (Hartree/Particle)

Thermal correction to Energy $=$

0.148713

Thermal correction to Enthalpy $=$ 0.149657

Thermal correction to Gibbs Free Energy $=0.093155$

Sum of electronic and zero-point Energies $=\quad-885.283181$ 


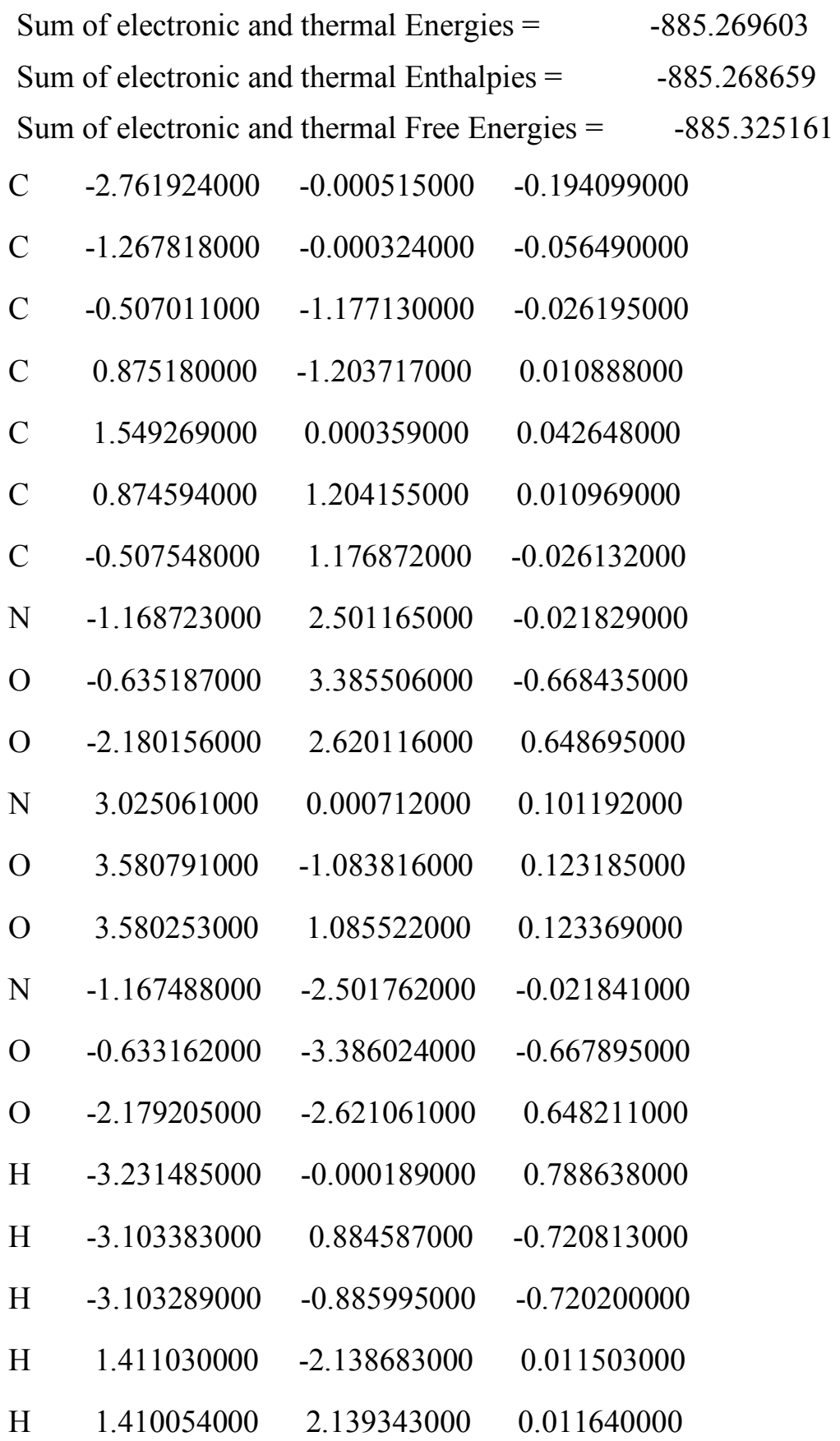

\section{HNB}

Number of Imaginary Frequencies $=0$

Zero-point correction=

0.112158 (Hartree/Particle)

Thermal correction to Energy=

0.132553

Thermal correction to Enthalpy=

0.133497

Thermal correction to Gibbs Free Energy=

0.061208

Sum of electronic and zero-point Energies=

$-1459.622175$

Sum of electronic and thermal Energies=

$-1459.601780$

Sum of electronic and thermal Enthalpies=

$-1459.600836$ 


$$
\begin{array}{lrrr}
\multicolumn{5}{c}{\text { Sum of electronic and thermal Free Energies }=} & -1459.673125 \\
\mathrm{C} & 0.551580000 & 1.269479000 & -0.000035000 \\
\mathrm{C} & -0.823614000 & 1.112453000 & 0.000044000 \\
\mathrm{C} & -1.375194000 & -0.157050000 & 0.000030000 \\
\mathrm{C} & -0.551599000 & -1.269489000 & 0.000013000 \\
\mathrm{C} & 0.823628000 & -1.112436000 & 0.000110000 \\
\mathrm{C} & 1.375203000 & 0.157038000 & 0.000062000 \\
\mathrm{~N} & 2.851595000 & 0.325705000 & 0.000136000 \\
\mathrm{O} & 3.444519000 & -0.219784000 & 0.904375000 \\
\mathrm{O} & 3.306154000 & 0.990833000 & -0.904213000 \\
\mathrm{~N} & -1.143782000 & -2.632392000 & -0.000216000 \\
\mathrm{O} & -1.913137000 & -2.873048000 & 0.903657000 \\
\mathrm{O} & -0.794562000 & -3.358699000 & -0.904279000 \\
\mathrm{~N} & -1.707859000 & 2.306730000 & 0.000086000 \\
\mathrm{O} & -2.511274000 & 2.367787000 & -0.904129000 \\
\mathrm{O} & -1.531893000 & 3.092892000 & 0.904397000 \\
\mathrm{~N} & 1.707873000 & -2.306690000 & 0.000135000 \\
\mathrm{O} & 2.511721000 & -2.367476000 & -0.903712000 \\
\mathrm{O} & 1.531300000 & -3.093300000 & 0.903964000 \\
\mathrm{~N} & -2.851595000 & -0.325643000 & 0.000091000 \\
\mathrm{O} & -3.306221000 & -0.991185000 & -0.903896000 \\
\mathrm{O} & -3.444453000 & 0.220290000 & 0.904141000 \\
\mathrm{~N} & 1.143785000 & 2.632353000 & -0.000176000 \\
\mathrm{O} & 1.913498000 & 2.872746000 & 0.903463000 \\
\mathrm{O} & 0.794331000 & 3.358893000 & -0.903986000 \\
\hline
\end{array}
$$

\section{TNB}

Number of Imaginary Frequencies $=0$

Zero-point correction= 0.107296 (Hartree/Particle)

Thermal correction to Energy=

0.119305

Thermal correction to Enthalpy=

0.120249

Thermal correction to Gibbs Free Energy=

0.066664

Sum of electronic and zero-point Energies= $-845.981327$

Sum of electronic and thermal Energies= $-845.969319$

Sum of electronic and thermal Enthalpies= $-845.968374$ 


$$
\begin{array}{lrrr}
\multicolumn{5}{c}{\text { Sum of electronic and thermal Free Energies }=} & -846.021959 \\
\mathrm{C} & 0.054163000 & -1.400371000 & 0.000048000 \\
\mathrm{C} & -1.155651000 & -0.728159000 & 0.000019000 \\
\mathrm{C} & -1.239840000 & 0.653281000 & -0.000021000 \\
\mathrm{C} & -0.052780000 & 1.364878000 & -0.000012000 \\
\mathrm{C} & 1.185683000 & 0.747077000 & 0.000007000 \\
\mathrm{C} & 1.208448000 & -0.636745000 & 0.000043000 \\
\mathrm{~N} & 2.519801000 & -1.327684000 & 0.000023000 \\
\mathrm{O} & 3.515799000 & -0.626591000 & 0.000047000 \\
\mathrm{O} & 2.504737000 & -2.545581000 & -0.000086000 \\
\mathrm{~N} & -0.110057000 & 2.846018000 & 0.000012000 \\
\mathrm{O} & -1.215232000 & 3.357962000 & -0.000040000 \\
\mathrm{O} & 0.952276000 & 3.441794000 & -0.000014000 \\
\mathrm{~N} & -2.409739000 & -1.518295000 & -0.000028000 \\
\mathrm{O} & -3.456883000 & -0.896187000 & 0.000089000 \\
\mathrm{O} & -2.300714000 & -2.731401000 & -0.000064000 \\
\mathrm{H} & -2.192995000 & 1.155501000 & -0.000068000 \\
\mathrm{H} & 2.097189000 & 1.321425000 & -0.000020000 \\
\mathrm{H} & 0.095787000 & -2.476928000 & 0.000088000
\end{array}
$$

\begin{tabular}{|c|c|c|c|c|}
\hline \multicolumn{3}{|c|}{ Zero-point correction $=$} & \multicolumn{2}{|c|}{0.154078 (Hartree/Particle) } \\
\hline \multicolumn{3}{|c|}{ Thermal correction to Energy= } & \multicolumn{2}{|c|}{0.171356} \\
\hline \multicolumn{3}{|c|}{ Thermal correction to Enthalpy= } & \multicolumn{2}{|c|}{0.172300} \\
\hline \multicolumn{4}{|c|}{ Thermal correction to Gibbs Free Energy= } & 0.107025 \\
\hline \multicolumn{4}{|c|}{ Sum of electronic and zero-point Energies= } & -1145.186526 \\
\hline \multicolumn{4}{|c|}{ Sum of electronic and thermal Energies $=$} & -1145.169249 \\
\hline \multicolumn{4}{|c|}{ Sum of electronic and thermal Enthalpies= } & -1145.168305 \\
\hline \multicolumn{4}{|c|}{ Sum of electronic and thermal Free Energies $=$} & -1145.233579 \\
\hline & 2.631300000 & -0.606764000 & \multicolumn{2}{|c|}{-1.479639000} \\
\hline & 1.911509000 & -0.367595000 & \multicolumn{2}{|c|}{-0.229197000} \\
\hline & 0.516157000 & -0.166939000 & \multicolumn{2}{|c|}{-0.187902000} \\
\hline & -0.351342000 & -1.260199000 & \multicolumn{2}{|c|}{-0.084525000} \\
\hline & -1.726218000 & -1.122704000 & \multicolumn{2}{|c|}{-0.010051000} \\
\hline
\end{tabular}

14. TETRL

Number of Imaginary Frequencies $=0$ 


$\begin{array}{lrrr}\mathrm{C} & -2.255633000 & 0.149882000 & -0.044102000 \\ \mathrm{C} & -1.452879000 & 1.273429000 & -0.108553000 \\ \mathrm{C} & -0.086412000 & 1.100733000 & -0.187971000 \\ \mathrm{~N} & 0.721575000 & 2.340073000 & -0.272344000 \\ \mathrm{O} & 0.266942000 & 3.325417000 & 0.272615000 \\ \mathrm{O} & 1.760400000 & 2.291219000 & -0.909642000 \\ \mathrm{~N} & -3.725004000 & 0.318653000 & 0.014493000 \\ \mathrm{O} & -4.396560000 & -0.696193000 & 0.067651000 \\ \mathrm{O} & -4.151809000 & 1.459118000 & 0.002673000 \\ \mathrm{~N} & 0.168043000 & -2.641763000 & -0.000257000 \\ \mathrm{O} & -0.384560000 & -3.374871000 & 0.798799000 \\ \mathrm{O} & 1.081861000 & -2.950373000 & -0.743611000 \\ \mathrm{~N} & 2.642551000 & 0.189724000 & 0.818664000 \\ \mathrm{O} & 3.846459000 & 0.195038000 & 0.687167000 \\ \mathrm{O} & 2.000383000 & 0.583798000 & 1.781306000 \\ \mathrm{H} & 3.090864000 & 0.316114000 & -1.825740000 \\ \mathrm{H} & 3.382605000 & -1.374174000 & -1.325766000 \\ \mathrm{H} & 1.906300000 & -0.961611000 & -2.203448000 \\ \mathrm{H} & -2.363692000 & -1.985912000 & 0.091552000 \\ \mathrm{H} & -1.881561000 & 2.262097000 & -0.095305000\end{array}$

\section{RDX}

Number of Imaginary Frequencies $=0$

Zero-point correction=

0.142356 (Hartree/Particle)

Thermal correction to Energy=

0.154876

Thermal correction to Enthalpy=

0.155821

Thermal correction to Gibbs Free Energy=

0.101153

Sum of electronic and zero-point Energies= $-897.646863$

Sum of electronic and thermal Energies= $-897.634343$

Sum of electronic and thermal Enthalpies= $-897.633399$

Sum of electronic and thermal Free Energies= $-897.688066$
$\begin{array}{lll}\text { C } & -0.385422000 & -1.367707000\end{array}$
1.177139000
$\mathrm{N} \quad-1.353462000 \quad-0.343695000$
0.814325000
$\begin{array}{lll}\text { C } & -0.991458000 & 1.017849000\end{array}$
1.178253000
$\begin{array}{lll}\mathrm{N} & 0.379441000 & 1.344832000\end{array}$
0.815748000 


$\begin{array}{lrrr}\mathrm{C} & 1.377254000 & 0.349856000 & 1.179189000 \\ \mathrm{~N} & 0.975195000 & -1.000337000 & 0.814817000 \\ \mathrm{~N} & 1.507772000 & -1.545607000 & -0.381700000 \\ \mathrm{O} & 2.510596000 & -1.018621000 & -0.815675000 \\ \mathrm{O} & 0.953018000 & -2.531759000 & -0.819346000 \\ \mathrm{~N} & 0.586396000 & 2.076972000 & -0.381939000 \\ \mathrm{O} & -0.371516000 & 2.679903000 & -0.818485000 \\ \mathrm{O} & 1.718471000 & 2.090349000 & -0.817729000 \\ \mathrm{~N} & -2.094283000 & -0.531308000 & -0.380738000 \\ \mathrm{O} & -2.138500000 & -1.662592000 & -0.816662000 \\ \mathrm{O} & -2.673234000 & 0.442101000 & -0.815565000 \\ \mathrm{H} & -0.414906000 & -1.472104000 & 2.261497000 \\ \mathrm{H} & -0.649647000 & -2.304597000 & 0.707520000 \\ \mathrm{H} & -1.670968000 & 1.715461000 & 0.709533000 \\ \mathrm{H} & -1.066721000 & 1.094539000 & 2.262702000 \\ \mathrm{H} & 2.321641000 & 0.589895000 & 0.711624000 \\ \mathrm{H} & 1.480257000 & 0.375763000 & 2.263747000\end{array}$

\section{HMX}

Number of Imaginary Frequencies $=0$

\begin{tabular}{|c|c|c|c|c|}
\hline \multicolumn{3}{|c|}{ Zero-point correction $=$} & \multicolumn{2}{|c|}{0.190639 (Hartree/Particle) } \\
\hline \multicolumn{3}{|c|}{ Thermal correction to Energy $=$} & & 08007 \\
\hline \multicolumn{3}{|c|}{ Thermal correction to Enthalpy= } & & 08951 \\
\hline \multicolumn{4}{|c|}{ Thermal correction to Gibbs Free Energy= } & 0.143351 \\
\hline \multicolumn{4}{|c|}{ Sum of electronic and zero-point Energies $=$} & -1196.863714 \\
\hline \multicolumn{4}{|c|}{ Sum of electronic and thermal Energies $=$} & -1196.846347 \\
\hline \multicolumn{4}{|c|}{ Sum of electronic and thermal Enthalpies= } & -1196.845402 \\
\hline \multicolumn{4}{|c|}{ Sum of electronic and thermal Free Energies $=$} & -1196.911003 \\
\hline $\mathrm{C}$ & -1.279328000 & 1.263367000 & 1.0732 & 95000 \\
\hline N & 0.000068000 & 1.515193000 & 0.4459 & 44000 \\
\hline $\mathrm{C}$ & 1.279358000 & 1.263312000 & 1.0733 & 63000 \\
\hline J & 1.882822000 & -0.000065000 & 0.7060 & 19000 \\
\hline & 1.279337000 & -1.263384000 & 1.0733 & 02000 \\
\hline & -0.000068000 & -1.515221000 & 0.4459 & 73000 \\
\hline & -1.279359000 & -1.263332000 & 1.0733 & 93000 \\
\hline
\end{tabular}




$\begin{array}{lrrr}\mathrm{N} & -1.882825000 & 0.000051000 & 0.706051000 \\ \mathrm{~N} & -3.202016000 & 0.000151000 & 0.220495000 \\ \mathrm{O} & -3.718725000 & -1.083988000 & 0.040291000 \\ \mathrm{O} & -3.718234000 & 1.084496000 & 0.040012000 \\ \mathrm{~N} & -0.000237000 & -1.875935000 & -0.901670000 \\ \mathrm{O} & 1.090099000 & -2.015470000 & -1.420840000 \\ \mathrm{O} & -1.090715000 & -2.015200000 & -1.420556000 \\ \mathrm{~N} & 3.202019000 & -0.000072000 & 0.220484000 \\ \mathrm{O} & 3.718573000 & 1.084143000 & 0.040298000 \\ \mathrm{O} & 3.718407000 & -1.084342000 & 0.040024000 \\ \mathrm{~N} & 0.000229000 & 1.875870000 & -0.901709000 \\ \mathrm{O} & -1.090111000 & 2.015336000 & -1.420890000 \\ \mathrm{O} & 1.090704000 & 2.015082000 & -1.420613000 \\ \mathrm{H} & -1.095417000 & 1.288025000 & 2.148955000 \\ \mathrm{H} & -1.983537000 & 2.041576000 & 0.807646000 \\ \mathrm{H} & 1.983625000 & 2.041468000 & 0.807796000 \\ \mathrm{H} & 1.095463000 & 1.287923000 & 2.149038000 \\ \mathrm{H} & 1.983539000 & -2.041600000 & 0.807667000 \\ \mathrm{H} & 1.095434000 & -1.288014000 & 2.148964000 \\ \mathrm{H} & -1.983623000 & -2.041492000 & 0.807840000 \\ \mathrm{H} & -1.095454000 & -1.287935000 & 2.149067000\end{array}$

\section{PETN}

Number of Imaginary Frequencies $=0$

Zero-point correction=

0.188814 (Hartree/Particle)

Thermal correction to Energy=

0.209502

Thermal correction to Enthalpy=

0.210446

Thermal correction to Gibbs Free Energy=

0.133674

Sum of electronic and zero-point Energies=

$-1316.844028$

Sum of electronic and thermal Energies=

$-1316.823340$

Sum of electronic and thermal Enthalpies=

$-1316.822396$

Sum of electronic and thermal Free Energies $=\quad-1316.899168$
$\begin{array}{llll}\text { C } & 0.000011000 & -1.227755000 & 0.922040000\end{array}$
$\begin{array}{llll}\text { C } & 0.000000000 & 0.000000000 & 0.000000000\end{array}$
$\begin{array}{llll}\text { C } & 1.227755000 & 0.000011000 & -0.922040000\end{array}$ 
